NETWORK TRAFFIC IMAGES

\title{
A DEEP LEARNING APPROACH TO THE CHALLENGE OF INTERNET TRAFFIC CLASSIFICATION
}

\author{
A Thesis \\ Presented to the \\ Faculty of \\ California State Polytechnic University, Pomona \\ In Partial Fulfillment \\ Of the Requirements for the Degree \\ Master of Science \\ In \\ Computer Science
}

By

Ibraheem Y. Saleh

2018 
SIGNATURE PAGE

THESIS:

AUTHOR:

DATE SUBMITTED:

Dr. Hao Ji

Thesis Committee Chair

Computer Science

Dr. Amar Raheja

Computer Science

Dr. Yu Sun

Computer Science
NETWORK TRAFFIC IMAGES: A DEEP LEARNING APPROACH TO THE CHALLENGE OF INTERNET TRAFFIC CLASSIFICATION

Ibraheem Y. Saleh

Fall 2018

Department of Computer Science 


\section{ACKNOWLEDGEMENTS}

The amount of thanks owed to the people in my life who have made this research possible is unable to be captured on one page.

At the most immediate level, I would like to thank the review committee, Dr. Hao Ji, Dr. Amar Raheja and Dr. Yu Sun for working with me in the short time-frames that I provided them with to complete this research effort.

I would like to especially thank Dr. Hao Ji for encouraging me to publish my first research paper over a year ago and exposing me to the world of conferences and peer review that I never knew existed before. His professional insights and teaching methods were the best that I experienced at Cal Poly Pomona.

I would also like to thank my colleagues at the Jet Propulsion Laboratory who have been extremely encouraging of my pursuit to obtain my Masters in Computer Science. Without their patience with me taking off for finals or to wrap-up large projects, especially this thesis, none of what I have accomplished over the past two years would have been possible.

Even more thanks and praise are owed to my dearest Mother who has been supportive and encouraging of all of my personal and professional pursuits in life. Without her, I can't imagine what kind of a disaster life would have made of me!

The most important of all praise and thanks goes to God, who has blessed me with a seemingly infinite number of blessings, some small and some massive, the sum total of which are the reason why everything I have accomplished, including this research, was possible. 


\begin{abstract}
The challenge of network traffic classification exists at the heart of many networking related tasks aimed at improving the overall user experience and usability of the internet. Traditional approaches to classifying network traffic, for example deep packet inspection techniques, rely on humans manually developing and maintaining application communication signatures, which among other shortcomings, can prove near impossible to keep updated given the rapid expansion in the number of different communicating applications. Current research-level approaches to the challenge of traffic classification that are more dynamic in nature due to the use of support vector machines and clustering technologies are, in some ways, superior to traditional methods but still fall short under varying usage scenarios. In this research, we introduce the concept of network traffic images, a reimagination of the 1-dimensional sequential packet stream as a 2-dimensional array of packet header lengths, and characterize the usefulness of these images for traffic classification when evaluated with modern deep learning technologies, namely convolutional neural networks. To the best of the authors knowledge, the concept of network traffic images and the utilization of deep learning techniques for the purposes of network traffic classification have never been attempted previously in any published research and are entirely novel. The experimental results of this new classification methodology are promising and demonstrate that there is significant potential for this technology to be adopted in real-world venues.
\end{abstract}




\section{TABLE OF CONTENTS}

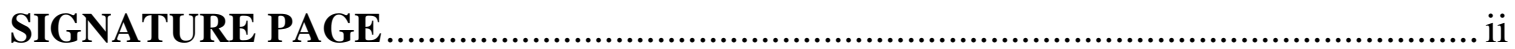

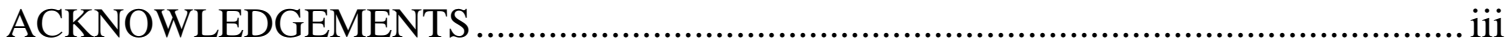

ABSTRACT

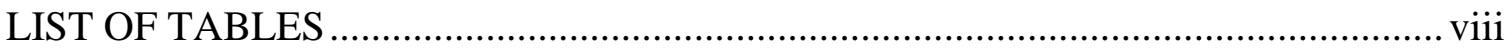

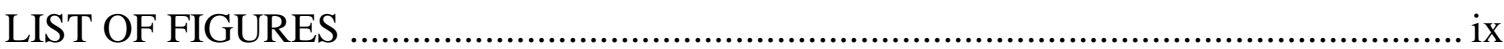

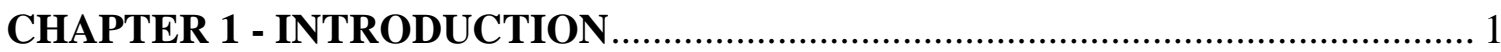

1.1 Significance and Problem ................................................................................ 1

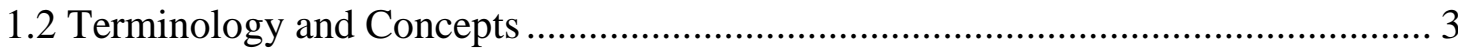

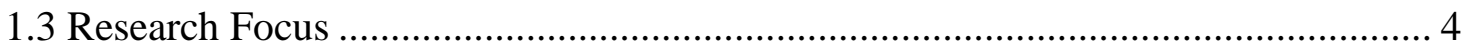

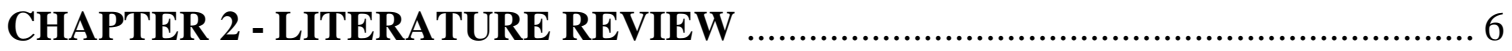

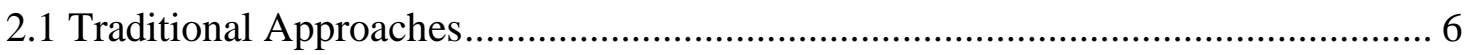

2.1.1 Port Mapping Based Traffic Classification...................................................... 6

2.1.2 Deep Packet Inspection (DPI) Based Traffic Classification .................................. 7

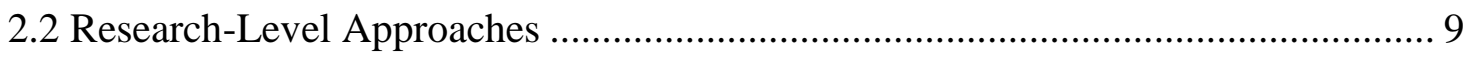

2.2.1 Host-Behavior Based Network Traffic Classification Techniques.................... 9

2.2.2 Packet Flow Feature Based Traffic Classification Techniques ....................... 11

2.3 Challenges in Comparing Different Research Efforts .......................................... 12

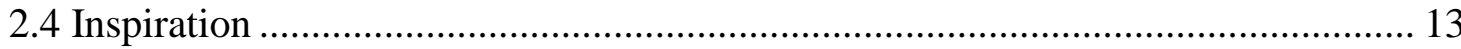




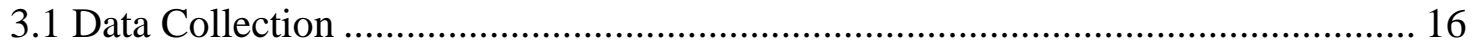

3.1.1 Data Organization Planning ........................................................................... 16

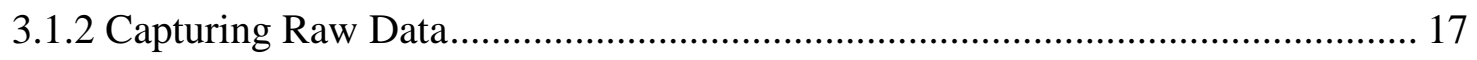

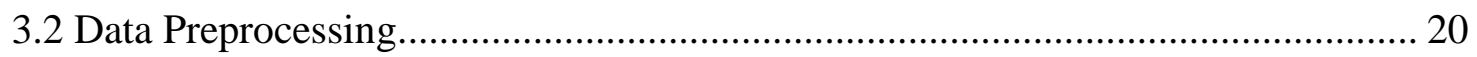

3.2.1 Separating Out Useful Connection Streams ………………............................ 20

3.2.2 Generating Network Traffic Images (NTIs) ………….................................. 21

3.2.2.1 Network Traffic Image Arrangement ........................................................ 22

3.2.2.1.1 Linear NTI Arrangement ................................................................... 22

3.2.2.1.2 Diagonal NTI Arrangement ……………………................................. 23

3.2.2.1.3 Waterfall NTI Arrangement................................................................... 24

3.2.2.1.4 Center Spiral NTI Arrangement.......................................................... 25

3.2.2.1.5 Edge Spiral NTI Arrangement ............................................................. 26

3.2.2.2 Packet-Relative Network Traffic Images..................................................... 27

3.2.2.3 Time-Relative Network Traffic Images....................................................... 28

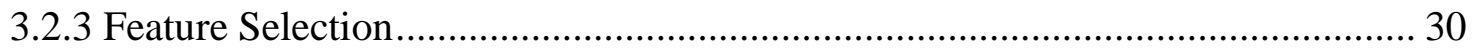

3.3 Machine Learning Model.................................................................................. 31

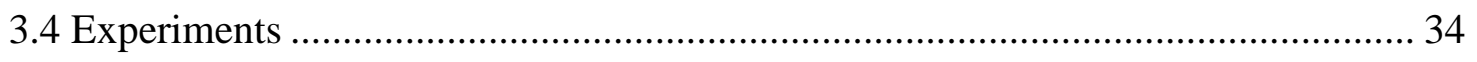

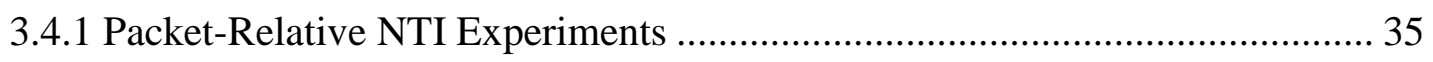


3.4.2 Time-Relative NTI Experiments.

4.1 Packet-Relative NTI Experiment Results ........................................................ 39

4.1.1 17x17 Packet-Relative NTI Results ............................................................. 39

4.1.2 49x49 Packet-Relative NTI Results ........................................................... 40

4.2 Time-Relative NTI Experiment Results ..................................................... 42

4.2.1 Time-Relative NTI Experiment Design Issue............................................. 42

4.2.2 17x17 5-second Time-Relative NTI with 10\% Fill Results.............................. 45

4.2.2 25x25 10-second Time-Relative NTI with 5\% Fill Threshold Results ............. 46

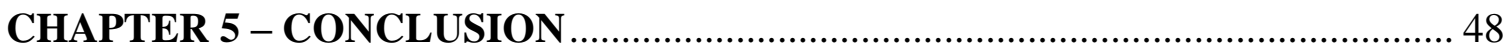

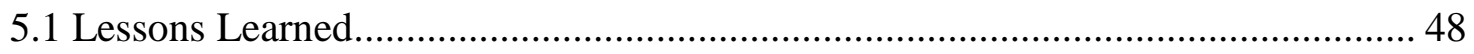

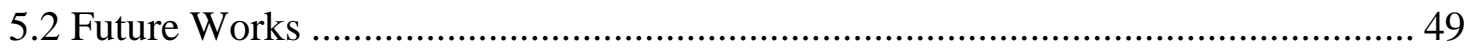

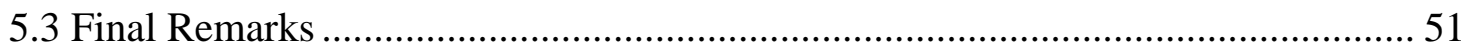

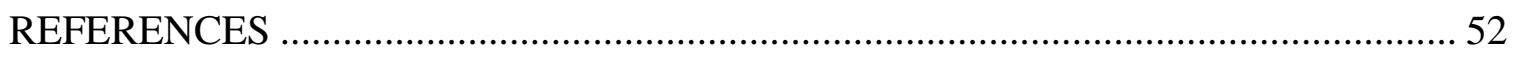

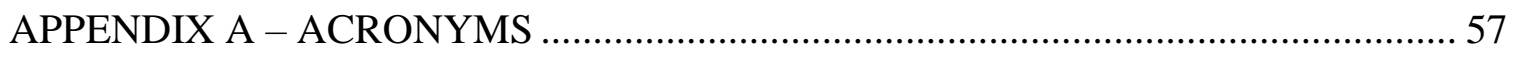




\section{LIST OF TABLES}

Table 1: Table of Applications and Classifiers used in this Research ........................... 17

Table 2:17x17 packet-relative NTI experimentation results ..................................... 39

Table 3: 49x49 packet-relative NTI experimentation results ..................................... 41

Table 4: 17x17 5-second Time relative NTI with forced 10\% fill results ..................... 45

Table 5: 25x25 10-second Time relative NTI with forced 5\% fill results ...................... 46 


\section{LIST OF FIGURES}

Figure 1: Host based traffic classification example (Left is torrent connection, right is

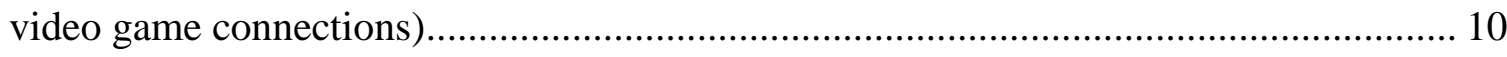

Figure 2: Experiment Preparation and Execution Flow Diagram to Calculate NTI

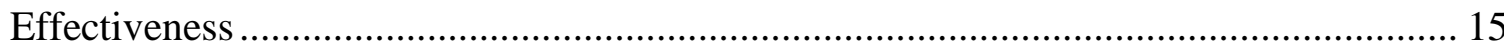

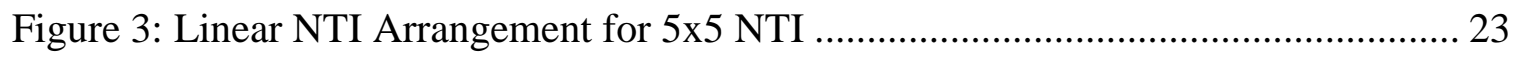

Figure 4: Diagonal NTI Arrangement for 5x5 NTI ................................................... 23

Figure 5: Aerial View of Stream [42] and Front View of Waterfall [43] in Nature......... 24

Figure 6: Waterfall NTI Arrangement for 5x5 NTI with force vectors to show fill pattern 25

Figure 7: Galaxy [40], Rose [41] and Fibonnacci Spirals in Nature [34]....................... 25

Figure 8: Center Spiral NTI Arrangement for 5x5 NTI with vectors to show fill pattern 26 Figure 9:Edge Spiral NTI Arrangement for 5x5 NTI with vectors to show fill pattern ... 26

Figure 10: A simple packet-relative linear NTI mapping diagram.................................. 27

Figure 11: Packet Stream to linear Time-Relative NTI mapping example diagram ........ 29

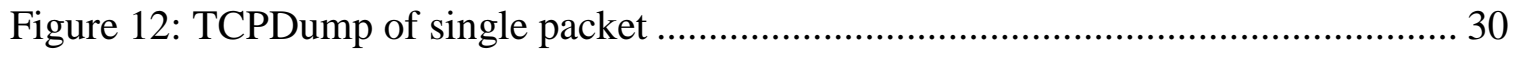

Figure 13: VGG-16 Model Variant Used as Starting Point for all NTI Experiments. ..... 33

Figure 14: Mindmap detailing all Packet-Relative NTI Experiments to be executed ...... 36

Figure 15:Mindmap detailing all Time-Relative NTI Experiments to be executed. ......... 38

Figure 16: 17x17 NTI Test Result Charts Plotted with Respect to Packet Arrangement. 40

Figure 17: 17x17 CSGO Time-Relative NTI with 1\% fill 43 
Figure 18: Mindmap showing the redesign of the Time-Relative NTI Experiments with

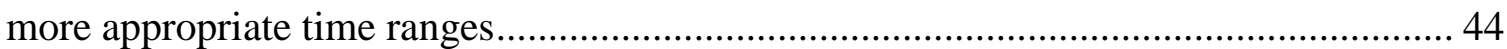




\section{CHAPTER 1 - INTRODUCTION}

\subsection{Significance and Problem}

Utilization of the internet by humanity as a whole has exhibited a steady upwards trend over the past twenty years [1]. Not only are the number of devices interconnected increasing at an exponential rate given, amongst other things, the explosion of internetof-things technology, but also, bandwidth utilization of computers is increasing on average as the quality and quantity of data rises. Our dependence on the successful communication of these devices naturally grows as they become more embedded in normal day-to-day operations.

According to Akamai's "State of the Internet” report in Q1 2017, the average connection speed in the United States of America hovers at around 18.7 Megabits per second (Mbps). [2] That same report postulates that the global average connection speed of internet users is around 7.2 Mbps. Given such limited internet connection speeds and continually growing bandwidth requirements for end-user applications, it doesn't take much for a network to experience bandwidth contention that can seriously cripple the usability of realtime-response applications such as video conferencing tools or entertainment services. Even in regions where internet connections speeds have reached rates of $100 \mathrm{Mbps}$ and above, where bandwidth pipeline limits are rarely reached, there still exists the problem where jitter and packet reception delays, caused by connection switching, can make applications that require the most rapid responses impacted and unusable.

To address this problem, quality of service software, usually embedded in the network routers themselves, perform network traffic classification so that traffic streams 
that demand realtime user responses can be prioritized over connections that are less quintessential in nature, such as http downloads or file transfers.

Network traffic classification is the science of ingesting a connection stream, or part of a connection stream, and classifying it in a meaningful way. Though there exist a plethora of ways in which traffic can be classified to be made useful in applications with different purposes, for example, a security oriented network traffic classifier might inspect network traffic and determine "threat" or "safe", the primary focus of this research is on the type of internet traffic classification utilized to assign connections to various classes of service which could then be prioritized by other networking components accordingly. For example, a simple quality of service focused traffic classifier might look at a given connection and decide that it belongs to one of three classes: "realtime-priority", "normal-priority" or "low-priority". This classification establishes a quality of service baseline that enables the limited bandwidth of any network to be utilized to its maximum potential by limiting less important, bandwidth hogging connections and prioritizing real-time response demanding applications.

A fully bandwidth saturated network behaves identically to having no network connection at all and causes a denial of service for people attempting to communicate over the internet. Even a moderately bandwidth saturated network can cause certain applications to be completely unusable. Accurate and efficient network traffic classification is critical to enabling network administrators to be able to ensure that the internet communication needs of people are always fulfilled. 


\subsection{Terminology and Concepts}

The following terminologies and concepts are essential to understanding this paper.

- Network Traffic Classification: The science of ingesting a connection stream, or part of a connection stream, and classifying it in a meaningful way. The goal of this research is to classify connections to accurately identify the class of service that each belong to, ideally, for use in a QoS engine.

- Quality of Service: Quality of service refers to the ability to provide network traffic prioritization with the intention of guaranteeing performance to certain high-priority connection types.

- Connection (network connection): A connection is defined on the router as the 5-tuple (source_ip, destination_ip, source_port, destination_port, protocol) over a specified tie period. [3] At any given time, each network connection in the public internet is unique.

- Packet (network packet): A packet is the medium of communication by which bytes of information are transferred between two hosts on each side of the connection.

- Connection Stream: A connection stream refers to the collection of network packets that encompass the communication within a connection.

- Packet Flow (packet stream): Interchangeable with connection stream-explicitly refers to the packet components of the connection stream. Every packet sent from a source host to a destination host over the same port are considered to be part of the same packet flow. 
- Packet Subflow: A packet subflow is a subset of a packet flow which only includes a small time-delimited range of packets instead of the entire packet stream.

- Zero-Day Application: An application whose network traffic has never before been seen by a traffic classification engine.

- Overfitting: A machine learning concept where a model fits so closely to the training data that it becomes incapable of accurately fitting to the testing data.

- Convolutional Neural Networks: A type of deep neural network designed and primary used with images that has had great success in multiple machine learning venues over the past decade.

\subsection{Research Focus}

In this research, we primarily investigate network traffic classification for the purposes of enabling quality of service engines to function accurately. We introduce the concept of a network traffic image (NTI), a 2-dimensional transformation of a packet subflow, and attempt to prove the usability of these NTIs by exercising them through a large number of telling experiments. The rest of this paper is formulated as follows:

Chapter 2 reviews some of the traditional approaches to network traffic classification, namely port mapping and deep packet inspection approaches, including why they are useful and their short-comings relative to a quality of service focused classification engine. Then-after, we discuss current research level approaches to network traffic classification describing how they work and where the variation between them generally occurs. Then, we mention the inspiration for our current research and some of the goals hither-to. 
Chapter 3 details the procedures involved in network traffic classification experiment planning and setup and explains the novel idea of network traffic images in detail, including what they are and how they are generated.

Chapter 4 explains the experimental results and explores the discoveries made throughout their execution in detail.

Chapter 5 concludes the paper, summarizing the experiments conducted and their results, and describes some of the potential future works that logically derive from the research presented in this work. 


\section{CHAPTER 2 - LITERATURE REVIEW}

The challenge of network traffic classification has been tackled by researchers in a multitude of ways that span as far back as the invention of network communication itself. Classification approaches can generally be broken down into two broad categories: Traditional approaches, which usually include port-mapping and deep packet inspection techniques and require some degree of manual effort to maintain, and current researchlevel approaches which normally involve various machine learning driven architectures to try and minimize the human element in accurately defining classification rules.

\subsection{Traditional Approaches}

Traditional approaches to network traffic classification generally include portmapping approaches and deep packet inspection (DPI) based approaches. They are traditional in that they require humans to manually maintain a collection of identifiers that are used to classify traffic and, as such, can be ineffective, expensive and or difficult to sustain.

\subsubsection{Port Mapping Based Traffic Classification}

Port mapping based classification is both the simplest and most efficient of the approaches to network traffic classification. Naturally, it also the most limited technique to classify traffic. It works by looking at the source and destination ports of a connection and performing a lookup of these ports from the Internet Assigned Numbers Authority's (IANA) database of port mapping. The results of this lookup are then used to classify the connection. [4] When companies develop new applications and standardize the port that they communicate over, they are expected to file a request with the IANA to add their new port to the existing IANA port mapping database. 
Port mapping was a fairly simple solution to traffic classification that worked quite well in the early days of the internet. However, now that the internet is so widespread, a number of problems arise with this method. The most basic problem is that the IP headers used to route internet traffic only support 16 bits for the port field. This means that there are only $2^{16}$, or 65536 , available unique ports to be mapped and there are well over that many applications that communicate publicly over the internet. In addition to this, a large number of applications, such as OpenVPN [5] which tunnels it's connections over port 443, a well recognized port for HTTPS website communication, masquerade their connections over widely used ports to avoid being blocked by network firewalls. There are more reasons why port mapping approaches are significantly flawed but the aforementioned are enough to know that it is far too ineffective for the purposes of internet traffic classification in the modern age.

\subsubsection{Deep Packet Inspection (DPI) Based Traffic Classification}

Deep packet inspection (DPI) based network traffic classification techniques are currently the most widely found solution implemented in commercial networking products such as the enterprise level SonicWall firewall line [6] and even consumer grade products such as the Ubiquity UniFI routers and firewalls [7]. The ntop company even provides an open source solution, nDPI, that is able to be executed on various Unix and Windows operating systems. [8]

Deep packet inspections works by looking at the application-layer content of packets in a packet stream and matching the packet signature to known application signatures. This collection of known application signatures is manually managed by the owners of the DPI software. When a new application is discovered that is of interest to 
DPI-based classification end users, software engineers will reverse engineer a packet stream from this application and tediously add this application's new signature to their collection of existing application signatures.

Since the DPI engine is examining the application layer bytes of a packet, it tends to be an extremely accurate network traffic classification solution. It is difficult for malicious applications to spoof their traffic signature to fool DPI engines. This makes DPI often utilized extensively in network security solutions.

Though DPI is a very effective traffic classification solution, there are a number of shortcomings with the technology that can't be overlooked. Firstly, by default, a DPI based classification engine is completely ineffective at classifying encrypted traffic since the application-layer data is obfuscated through encryption and won't match the predefined application signatures. To combat this, corporations employ SSL-DPI which decrypts and re-encrypts the network traffic before having it reach the targeted end-user. Not only is this an extremely resource intensive procedure, it is also a clear violation of the privacy intended by using secure communications in the first place. It is important to note that SSL-DPI only works with certificate based HTTPS traffic and remains completely ineffective against other encryption based tunneling technologies.

In addition to encrypted traffic concerns with DPI, there remains the serious problem of manpower that must be dedicated to adding new application packet signatures and updating existing ones when they change. On the open source nDPI website, the authors plead, "DPI is a time-consuming activity as protocols (in particular P2P) change quite often. This means that it's necessary to update the code from time to time and add extensions. We would encourage anyone out there to help us adding or enhancing new 
protocols: we will put your contributions on our SVN and make them available to everyone free of charge." [8]

Deep packet inspection is widely used and extremely accurate when it works. However, its ineffectiveness at classifying encrypted traffic, computational complexity and the difficulty to maintain up-to-date application signatures leaves much to be desired with regards to a state of the art network traffic classification engine.

\subsection{Research-Level Approaches}

Research-level approaches are titled as such since they are exactly that: researchlevel. These approaches have been formulated and tested mostly in sand-boxes and research labs and are extremely difficult to reproduce for a multitude of reasons. Each published approach to network traffic classification, however, is enlightening and expands the field ever so slightly. Each methodology generally aims at achieving high classifications accuracy while minimizing the human involvement in maintaining the classifiers.

Research-level approaches can further be broken down into two categories, hostbehavior based techniques and packet flow based techniques, including supervised and unsupervised methods. [3]

\subsubsection{Host-Behavior Based Network Traffic Classification Techniques}

Host-behavior based network traffic classification techniques make the assumption that the connection patterns between source and destination hosts are unique enough to function as a discriminating factor in traffic classification without any packet

level inspection done. The most prominent example of host-behavior based classification is with BLINC, an acronym for "BLINd Classification" introduced in [9]. In their 
research, Karagiannis et al develop their methodology with the assumed constraints that no access to user payloads is possible and that only the information from traffic flow collectors is available. They break their collected packet traces using three different types of descriptors, social level descriptors, functional level descriptors, and application level descriptors. For the social descriptors features, they determine the number of other hosts that a host communicates with. [9] achieves fairly good results for classifying connections of type p2p, web, ftp, mail, chat, and network management. Similarly, in [10], Bermoulen et al utilize number of peers per network along with other connection information features fed through a SVM to classify peer-to-peer real-time video streaming services and get decent results.

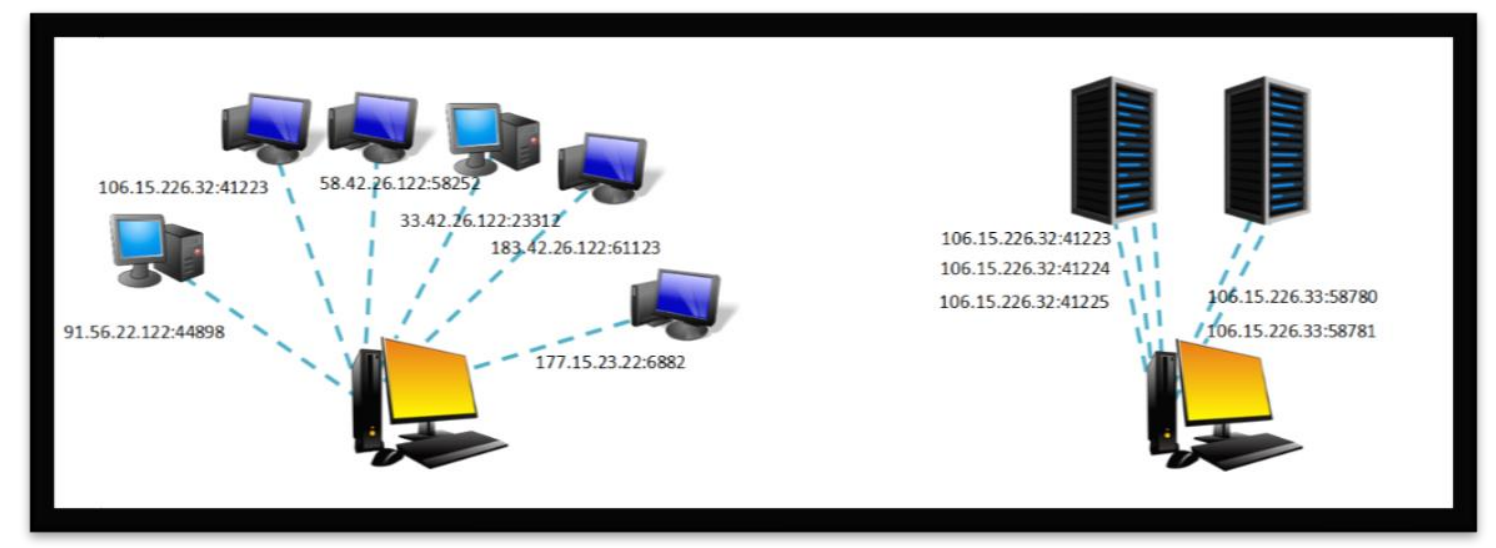

Figure 1: Host based traffic classification example (Left is torrent connection, right is video game connections)

Despite the seemingly promising results and the ingenuity of host-behavior based traffic classification, there are a number of short-comings that prevent it from every really being used in a quality-of-service type engine. The most crippling factor for this type of traffic classification is that there is no way for network hardware at the router level to determine which recently opened connections should be classified independently of each 
other--IE with regards to which application opened each connection. For example, if somebody starts a VoIP call at the same time that their operating system starts downloading a massive operating system patch, the classifier would certainly group all the connections just opened and send them through the BLIND classifier together and, since the connections would predominantly reflect a $\mathrm{p} 2 \mathrm{p}$ stream, the VoIP connection would be classified incorrectly. Though host-behavior based classifiers function well as simple, external network traffic monitoring tools, this type of traffic classification will likely never be realized in networked-managed hardware such as routers and QoS engines that do not have the crippling limitations assumed in those research efforts.

\subsubsection{Packet Flow Feature Based Traffic Classification Techniques}

Packet flow feature based traffic classification, unlike host-behavior classification, assumes that the classification engine has direct access to packet streams and statistical connection information. Instead of looking at the application level payload, as required by deep packet inspection techniques, flow-feature techniques capture flow information relative to each unique connection packet stream and then feed them through various different machine learning models to get varying degrees of classification accuracy.

The amount of features that can be derived and used for this type of classification is reported to be as high as 248 as enumerated in [11]. In [12], Moore et al use a Naive Bayesian estimator to exhaustively and automatically whittle down this list to determine which are the best for use for traffic classification purposes. Using hand classified data, they determine that the following twelve-features are most useful for classifying traffic: number of pushed data packets from the server to the client, initial window bytes from 
the client to the server, initial window bytes from the server to the client, the average segment size from the server to the client, the median of the IP data bytes from the client to the server, the number of data packets from the client to the server, the data bytes in the wire variance from the server to the client, the minimum segment size from the client to the server, the round-trip-time sample size from the client to the server and the number of pushed data packets from the client to the server. [12] Using just this subset of features, they claim that they are able to achieve over $95 \%$ accuracy for known traffic types. In [13], Jing et al utilize those same twelve features and propose a novel "fuzzy tournament mode SVM" model and achieve an average of 94\% accuracy with better computational performance than the model provided in [12]. The discovery of the power of packet flows for traffic classification has opened a world of research into their potential applications and increasing their classification accuracy. Some scientists have realized the power of packet flows and done research on classifying network traffic just using packet sub-flows, a subset of the entire packet flow. [3] Various models have been exercised including unique variations of SVMs, naive-bayesian models, boost models and more. Even unsupervised clustering models have been attempted that include kNN, kmeans, and DBSCAN, to name a few. Though the accuracies reported are specific to the domain studied and datasets utilized, the average accuracies that appear to most closely attempt what this research attempts, traffic classification of a packet sub flow using network traffic images, appears to fall within 73\%-91\% [3].

\subsection{Challenges in Comparing Different Research Efforts}

After reading through the research efforts of numerous other scientists who attempted to tackle the problem of network traffic classification of packet sub-flows, I 
quickly learned that it is almost impossible to compare the experimental results of any two research papers published in the field. Unlike image processing related efforts, which have sources such as the ImageNet [14] collection from Stanford and the KITTI [15] vision benchmark datasets with well laid out training and testing data collections with pre-defined targets, network traffic classification public datasets are much less mature. The lack of dataset maturity forces scientists to resort to using proprietary, manually collected datasets that are then almost never released for public experimentation. Of the 33 traffic classification papers surveyed in [3], 88\% of them ended up creating and using proprietary datasets.

Beyond the challenge of little to no dataset homogeneity, almost every study researched is unique in their targeted investigation goals. Even the papers that decide to exclusively compare class-of-service QoS related goals generally define the classes of importance vastly differently from each other. [3] This means that directly comparing each research-effort's reported accuracy ratings is generally not truly meaningful or representative of anything.

Until the aforementioned two problems are addressed, the majority of network traffic classification efforts will remain incomparable and not immediately applicable to real world classification solutions.

\subsection{Inspiration}

All of the research efforts that I discovered and read through utilized supervised support vector machines or various naive-bayes based classifiers, unsupervised clustering algorithms or other, related shallow learning approaches. There wasn't a single published research effort that utilized convolutional neural networks to attempt network traffic 
classification. There have been great strides forward in large-scale image and video recognition over the past few years particularly due to the power of deep convolutional neural networks. [16] In research that I have previously conducted, it was discovered that even for sound wave classification, convolutional neural networks, when fed raw spectrograms, outperform support vector machines and simple deep neural networks that are fed sound wave quantifying statistics from the same sources. [17] It is the primary goal of this work to introduce the power and success of convolutional neural networks to the field of network traffic classification through the invention of network traffic images. 


\section{CHAPTER 3 - METHODOLOGY AND EXPERIMENTS}

In order to successfully conduct this research and discover the effectiveness of deep learning with network traffic images (explained in detail below) for the purposes of network traffic classification, a number of preparatory steps must be executed.

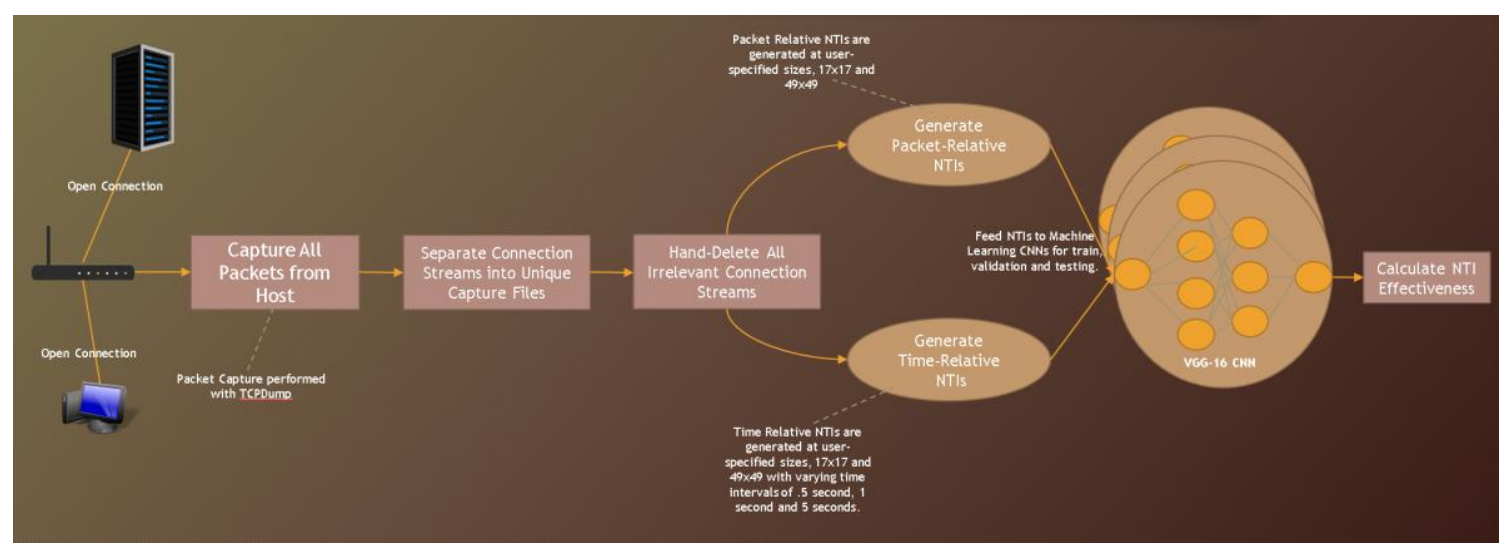

Figure 2: Experiment Preparation and Execution Flow Diagram to Calculate NTI Effectiveness

First, we must collect the raw data--the raw packet streams from the host that is

executing the application that will exercise the desired connection. Then we must separate these massive raw capture files into a collection of smaller capture files organized by the connections, the packet flows, that each captured packet belongs to. Then we manually delete all of the irrelevant packet streams so that only connections that we intend to classify remain. Then after, when we are left with just good packet streams belonging to the applications of interest, we organize those connections into either our train, test or zero-day connection subsets and generate all of the unique network traffic images that can possibly be generated from it. Once we have enough unique NTIs for an experiment, we train, validate and test our machine learning model to calculate the effectiveness of the NTI. 


\subsection{Data Collection}

The intial step in preparing our experiments is the dataset collection. Before we start capturing raw data, we first have to design and understand the needs of our experiments. Then, once we understand the goals of our classifier, we can capture and organize the raw data needed to support it.

\subsubsection{Data Organization Planning}

Before you can begin to collect the data to be used for network traffic classification purposes, you must decide which kinds of network traffic you intend to be classified by the classifier. For this research, we seek to build three different classifiers, each with varying degrees of classification complexity to determine the effectiveness of our network traffic images. The three classification classes are as follows:

1. Bulk vs All: The simplest classification type. It attempts to classify each connection stream as either being bulk_download (torrents or large HTTP downloads) or not being bulk_download (including video_conferencing applications, games, and streams)

2. QoS Based: A slightly more complex classification that has three different classification classes: "high priority"-which includes video_conferencing and game applications, "medium priority"-which includes live video streams and "lowest priority" which includes torrents and bulk_download applications.

3. Application Class Based: The most complex classification system attempted in this research. It attempts to classify traffic as 1 of 5 different classes: "video_conferencing", "games", "streams", "bulk_download" and "torrents". 
Since the "bulk vs all" and "QoS based" classifiers are simplifications of the "application class based" classifier, as long as we capture traffic separated by application classes, we can easily reorder the traffic to work for the other, simpler classifiers after the fact.

Once we know the classes of application that we seek to classify, we must then actually decide which real-world applications fall under these classes. The applications and classes used for this research are diagrammed in the table below.

Table 1: Table of Applications and Classifiers used in this Research

\begin{tabular}{|c|c|c|c|c|}
\hline \multicolumn{3}{|c|}{ Classifiers } & \multirow{2}{*}{ Applications } & \multirow{2}{*}{$\begin{array}{c}\text { Zero-Day } \\
\text { Applications }\end{array}$} \\
\hline Application Class & QoS & Bulk vs All & & \\
\hline Video_conferencing & \multirow[t]{2}{*}{ High Priority } & \multirow{3}{*}{ Not Bulk } & $\begin{array}{l}\text { Skype [18], } \\
\text { Webex [19] }\end{array}$ & Join.me [20] \\
\hline Games & & & $\begin{array}{c}\text { HoN }[21], \text { CSGO } \\
{[22]}\end{array}$ & DoTA 2 [23] \\
\hline Streams & $\begin{array}{l}\text { Medium } \\
\text { Priority }\end{array}$ & & $\begin{array}{c}\text { Twitch.tv [24], } \\
\text { Netflix [25] }\end{array}$ & $\begin{array}{c}\text { Youtube live } \\
{[26]}\end{array}$ \\
\hline Bulk_download & \multirow[t]{2}{*}{ Lowest Priority } & \multirow[t]{2}{*}{ Bulk } & $\begin{array}{l}\text { Steam [27], } \\
\text { Origin [28] }\end{array}$ & Battle.net [29] \\
\hline Torrent & & & Tixati [30] & UTorrent [31] \\
\hline
\end{tabular}

\subsubsection{Capturing Raw Data}

Since the focus of this research is to develop a methodology that can ultimately be used in a QoS engine that would exist within networking infrastructure devices, we capture network traffic at the router level: The access point that must be navigated through by any two client machines wishing to communicate over the internet.

On the router device, for this research, a PFsense firewall [32] was used as the routing software running on an Intel Celeron E3865U with 16GB of RAM and a 512GB 
SSD, we installed and configured TCPDump with LibPCap [33] to capture packets. To capture packets, TCPDump was executed as follows:

tcpdump -B 524288 -tttt -s 0 -vvv -i em1 "dst host 10.1.1.17 or src host 10.1.1.17"

>webex_raw_capture15.cap

The capture options specified are explained as follows:

- The '-B' option expands the default tcpdump buffer size so that less packets are dropped when transfer rates exceed what libpcap can support

- The '-tttt' option prints the timestamps of each packet captured as hours, minutes, seconds, and fractions of a second

- The '-s 0' option removes the default snapshot length captures and lets it be any value

- The '-vvv' option enables more verbosity in the output

- The '-i em1' option specifies which network interface tcpdump should listen on

- The "dst host 10.1.1.17 or src host 10.1.1.7" option adds a filter to instruct tcpdump to only capture packets going to or coming from the specified host -- the host that will be executing the application that we wish to capture packets for.

- The "> webex_raw_capture15.cap" option instructs tcpdump to output all of the packet capture information to the specified capture file -- This example file is named webex_raw_capture15.cap since it is intended to be filled with packets from webex and is the 15th capture that we have conducted so far.

This tcpdump command, in essence, captures all packets' statistics information originating to or coming from the specified host with information that is meaningful to this research. 
Once we execute this tcpdump command, we wait a minute and then execute the following command to log a timestamp for the capture that will be used later: echo 'date +"\%Y-\%M-\%d\%H:\%M:\%S.\%s" >> webex_raw_capture15.date We then migrate to our capture host that, in this case, will be executing the Webex application for the capture and launch a Webex connection for a moderate duration. After the Webex connection finishes, we execute the same command as before on the router: echo `date +"\%Y-\%M-\%d\%H:\%M:\%S.\%s">> webex_raw_capture15.date Then, we wait yet another minute and shut-down the tcpdump capture utility. We have effectively captured a raw packet stream using tcpdump that, among other things, includes atleast 1 Webex connection of interest within the date range previously logged. This will be further pre-processed later to properly separate out the Webex connection stream of interest from the other irrelevant packet flows. We proceed to do this for all the other applications that we desire packet captures for until we are satisfied with the amount of data collected and believe that it will be enough for our intended research goals. By the end of this capturing process, 15GB of packet statistics were gathered for various applications. Note, this is $15 \mathrm{~GB}$ of text files, where each 2 line-pair represent statistics for a single packet captured, not $15 \mathrm{~GB}$ of raw transfer bytes. IE:

2018-11-26 20:37:28.813555 IP (tos 0x10, ttl 64, id 0, offset 0, flags [DF], proto TCP (6), length 184)

ShadowSense.shadowdomain.ssh > Shadowscout.shadowdomain.57351: Flags [P.], cksum 0x16be (incorrect -> Oxd598), seq 1071693831:1071693975, ack 3718173606, win 513, length 144

The amount of raw data processed from these captures is likely in the hundreds of gigabytes in size. Occasionally, when a tcpdump packet capture is shutdown, you might run into output as follows:

- 3652182 packets captured 
- 4565711 packets received by filter

- 431367 packets dropped by kernel

Packets are sometimes dropped when the transfer rates of a connection are so fast that LibPCap cannot keep up even with the increased buffer size. The above example was the result of a Steam bulk_download capture with download rates of $\sim 10$ megabytes per second. This packet loss for extremely high rate applications is accepted as an unavoidable reality that is expected in the real world and that our machine learning models should account for when classifying network traffic.

\subsection{Data Preprocessing}

Once we have captured all of the network packet streams necessary for this research, we still have a significant amount of preprocessing before we can use the data in a meaningful way with our machine learning models. First we must separate out each unique connection stream, then manually remove any connection streams that don't make sense, and then lastly transform the remaining-good connection streams into the input for our machine learning models: network traffic images (NTIs).

\subsubsection{Separating Out Useful Connection Streams}

The data collection stage has left us with a large number of massive packet capture files each generally being comprised of thousands of unique connections which must be filtered through to remove those which are meaningless or invalid for our purposes. To do this, we must first parse through each packet capture file and break it down into a collection of sub-files corresponding to each unique packet flow. While we parse this file and create the derivative packet flows, we maintain a 'connection.map' mapping file which keeps track of the number of connections in each connection stream, 
the first packet received time for a connection stream, the last packet received time for a connection stream, the total number of packets in a stream and the total number of bytes for the entire stream.

Once we have completed separation and creation of the mapping file, we then go through the mapping file and, using the *.date files generated in the data collection phase, delete any packet flows with packet start times before the start date timestamp, any packet flows with packet start times after the end date time stamp and any packet flows with a number of packets less than a specified threshold (the smallest NTI to be tested). This allows us to automatically weed out a large number of the connections that have nothing to do with the targeted application of interest.

The remaining connections are then manually analyzed using their packet statistics and the ones that make sense for the application being captured are kept while the rest are discarded. This leaves us with only good packet flows that are pertinent to the connection types we seek to classify that can then be further transformed into network traffic images.

\subsubsection{Generating Network Traffic Images (NTIs)}

The core idea introduced in this research is that of network traffic images (NTIs). A network traffic image is a 2-Dimensional interpretation of a packet subflow designed to enable deep learning convolutional neural network $(\mathrm{CNN})$ machine learning models to be able to uniformly process packet sub-flows.

Unlike the spectrograms utilized in [17] and normal images captured from cameras, network traffic images are not a naturally occurring phenomena. The most natural representation for a packet stream is a 1-Dimensional time-ordered array where 
the earliest received packet for a connection is at the front and the last received packet for that same connection is at the tail end of the array. This form, however, is not conducive to $\mathrm{CNN}$ related machine learning methods.

There are two components central to constructing each NTI: Firstly, the NTI arrangement, responsible for determining where and how to fill the content of the NTI, and secondly the NTI fill subject which delineates exactly what content to fill each prearranged NTI block with. For this research, the subject can be either packet-relativity or time-relativity.

\subsubsection{Network Traffic Image Arrangement}

When a camera transforms our 3-dimensional world into a 2-dimensional image plane, it does so by making the size of each 2-D object relative to the depth in the 3D world. The NTI transformation is different in that we transform a 1-D world into a 2-D image plane. Since there is no seemingly innate transformation that makes sense for this, we must brute force various transformation patterns and experimentally determine if they are effective for the purposes of traffic classification. Even though a network traffic image is seemingly not a natural representative of network communication, there is seemingly still benefit in turning to nature for guidance in constructing NTIs. As such, this research will evaluate 5 unique NTI arrangement patterns, 3 of which are inspired by nature: Linear, Diagonal, Waterfall, Center Spiral and Edge Spiral.

\subsection{Linear NTI Arrangement}

The linear NTI arrangement is the most simplistic transformation of the packet stream into an NTI. For each NTI block, $\mathrm{n}_{1}, \mathrm{n}_{2} \ldots \mathrm{n}_{\mathrm{n}}$, in NTI N, equivalent to a pixel in an 
image plane, fill the NTI using a row-major ordering. For example, a $5 \times 5$ linear NTI would be arranged as follows:

\begin{tabular}{|c|c|c|c|c|}
\hline $\mathbf{n 1}$ & $\mathbf{n 2}$ & $\mathbf{n 3}$ & $\mathbf{n 4}$ & $\mathbf{n 5}$ \\
\hline $\mathbf{n 6}$ & $\mathbf{n 7}$ & $\mathbf{n 8}$ & $\mathbf{n 9}$ & $\mathbf{n 1 0}$ \\
\hline $\mathbf{n 1 1}$ & $\mathbf{n 1 2}$ & $\mathbf{n 1 3}$ & $\mathbf{n 1 4}$ & $\mathbf{n 1 5}$ \\
\hline $\mathbf{n 1 6}$ & $\mathbf{n 1 7}$ & $\mathbf{n 1 8}$ & $\mathbf{n 1 9}$ & $\mathbf{n 2 0}$ \\
\hline $\mathbf{n 2 1}$ & $\mathbf{n 2 2}$ & $\mathbf{n 2 3}$ & $\mathbf{n 2 4}$ & $\mathbf{n 2 5}$ \\
Figure 3: Linear NTI Arrangement for $5 \times 5$ NTI
\end{tabular}

Though in many ways the linear arrangement is similar to the linear packet stream, we suspect that the seemingly arbitrary relationships between the blocks of each row in the NTI, for example between $n 1$ and $n 6$ or $n 7$, will cause features that have no real meaning to be learning by our convolutional neural network models.

\subsection{Diagonal NTI Arrangement}

The diagonal NTI arrangement is also a fairly simplistic transformation. For each NTI block, $\mathrm{n}_{1}, \mathrm{n}_{2} \ldots \mathrm{n}_{\mathrm{n}}$, in NTI N, arrange the blocks diagonally from the bottom up starting from the origin at the top left corner of the NTI. For example, a 5x5 diagnoal NTI would be arranged as follows:

\begin{tabular}{|c|c|c|c|c|}
\hline $\mathbf{n 1}$ & $\mathbf{n 3}$ & $\mathbf{n 6}$ & $\mathbf{n 1 0}$ & $\mathbf{n 1 5}$ \\
\hline $\mathbf{n 2}$ & $\mathbf{n 5}$ & $\mathbf{n 9}$ & $\mathbf{n 1 4}$ & $\mathbf{n 1 9}$ \\
\hline $\mathbf{n 4}$ & $\mathbf{n 8}$ & $\mathbf{n 1 3}$ & $\mathbf{n 1 8}$ & $\mathbf{n 2 2}$ \\
\hline $\mathbf{n 7}$ & $\mathbf{n 1 2}$ & $\mathbf{n 1 7}$ & $\mathbf{n 2 1}$ & $\mathbf{n 2 4}$ \\
\hline $\mathbf{n 1 1}$ & $\mathbf{n 1 6}$ & $\mathbf{n 2 0}$ & $\mathbf{n 2 3}$ & $\mathbf{n 2 5}$ \\
\hline
\end{tabular}

In many ways, the diagonal NTI arrangement is similar to that of the linear NTI arrangement, however, the proximity between neighbors in a diagonal NTI should be slightly closer to the proximity found in the 1-D packet stream. For example, in the 
diagonal arrangement, $\mathrm{n} 1$ is much closer to $\mathrm{n} 2$ and $\mathrm{n} 3$ than when linearly arranged. Since, in most instances, the proximities are closer to that found in the 1-D packet stream, we suspect this arrangement will perform superior to the linear arrangement.

\subsection{Waterfall NTI Arrangement}

The waterfall NTI arrangement is inspired by nature. If you look at an aerial view of a stream before it heads over a cliff, in many ways, it closely represents the natural form of a connection stream in that it appears to be 1-Dimensional and has a source and a destination with which content is transferred. Once a stream reaches the face of a cliff, it undergoes a transformation from a 1-D stream into a seemingly 2-D waterfall due to the forces of gravity mixed with the forces of the current pulling it downwards and outwards.
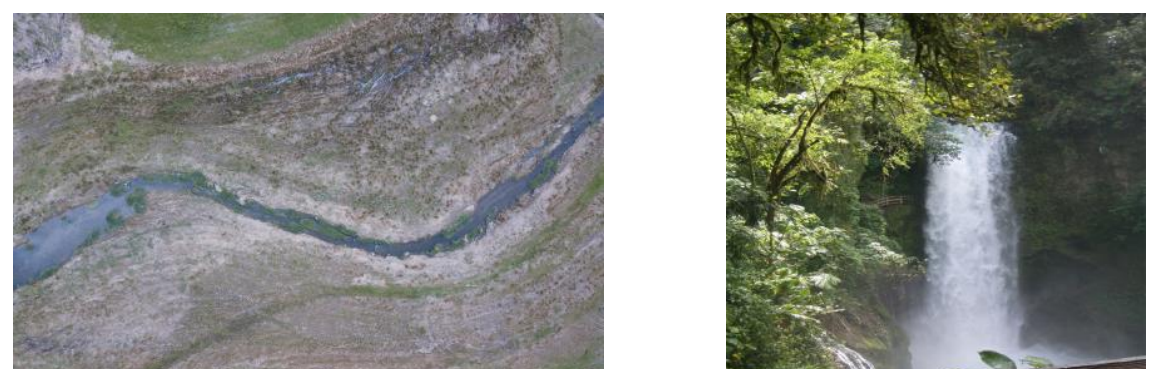

Figure 5: Aerial View of Stream [42] and Front View of Waterfall [43] in Nature

We attempt to imitate the same forces observed in a waterfall in the construction of our waterfall NTI. For simplicity, we still start the filling the NTI from the first position in the 2D NTI array and act as though gravity exists in the final row column position $\left(\mathrm{N}_{\mathrm{x}}, \mathrm{N}_{\mathrm{y}}\right)$, pulling it downwards first and then outwards sequentially in each direction. For example, a 5x5 waterfall NTI would be arranged as follows: 


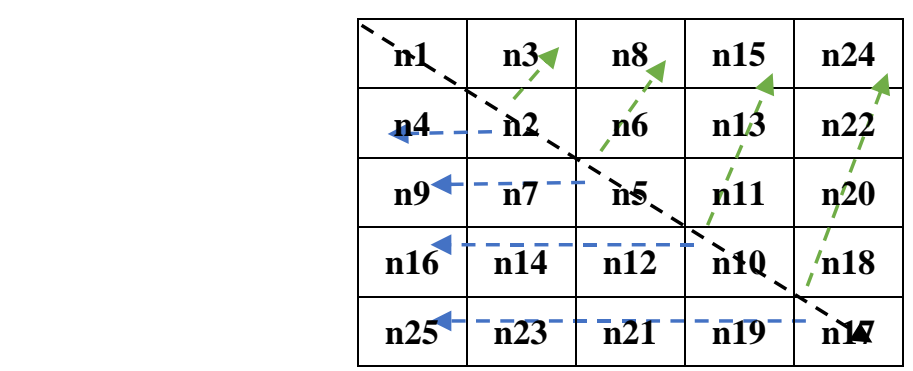

Figure 6: Waterfall NTI Arrangement for $5 \times 5$ NTI with force vectors to show fill pattern

Given the inspiration for the waterfall NTI design and the completely unique fill pattern that most closely represents the $1 \mathrm{D}$ proximities, we suspect that this will be a superior packet arrangement pattern.

\subsection{Center Spiral NTI Arrangement}

Similar to the waterfall pattern, the center spiral pattern is also nature-inspired. If we zoom out our perspective of the world millions of lightyears in distance and take a snapshot of our milky-way galaxy, it appears to us to be arranged in a spiral. If we zoom in and look at the structure of a rose, we also find this same majestic spiral form.

Scientists have discovered that if you zoom even further in yet, you can see spirals, namely fibonacci spirals, on conical-shaped microstructures. [34]
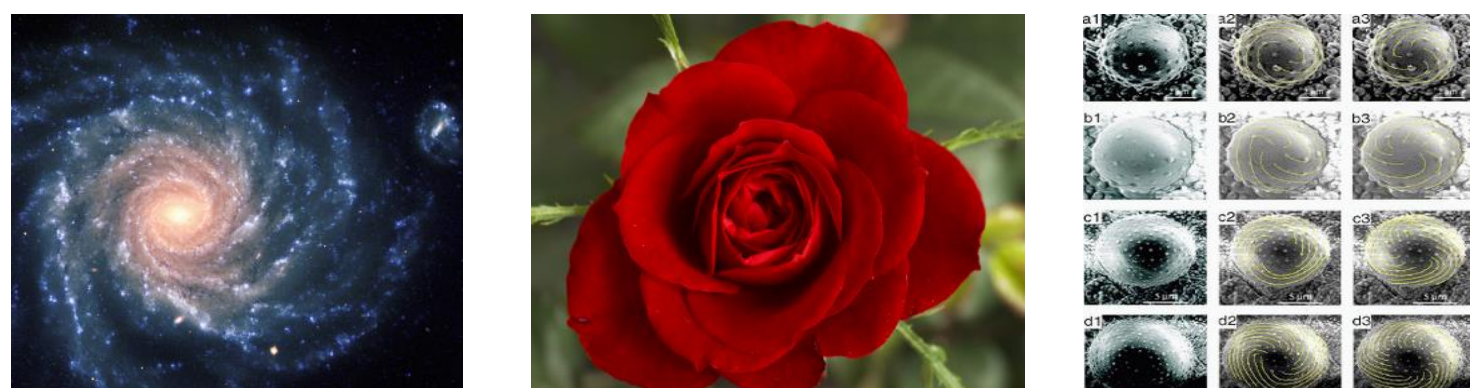

Figure 7: Galaxy [40], Rose [41] and Fibonnacci Spirals in Nature [34]

To fill an NTI with a center spiral arrangement, we start at the center position of the NTI and slowly loop around each layer of the spiral in a sequential manner. The number of loop-iterations required to fill an NTI is equal to $\min \left(\mathrm{N}_{\mathrm{x}}, \mathrm{N}_{\mathrm{y}}\right) / 2$, the minimum of the specified $\mathrm{x}$ and $\mathrm{y}$ dimensions of the NTI divided by two. The direction first 
expanded upon from the center point is arbitrary since a center spiral is symmetrical in shape. For example, a 5x5 center spiral NTI would be arranged as follows:

\begin{tabular}{|c|c|c|c|c|}
\hline $\mathrm{n} 25$ & $\overline{\mathrm{n} 24}$ & $\overline{\mathbf{n}} \overline{\mathbf{3}}$ & $\overline{\mathrm{n} 22}$ & $\bar{n} \overline{2} \overline{1}_{1}$ \\
\hline n10 & $\overline{n 9}^{-1}$ & $\overline{\mathrm{n}} \overline{8}$ & $\overline{n 7} \overline{4}$ & n20! \\
\hline n11 & n2 & - $\underline{n} 10$ & n6 & \\
\hline n12 & n3 $\frac{1}{2}$ & n4 & n5 & n18! \\
\hline n13 & 14 & $n 15$ & n16 & $\underline{n} 17^{\prime}$ \\
\hline
\end{tabular}

Figure 8: Center Spiral NTI Arrangement for $5 \times 5$ NTI with vectors to show fill pattern

In a center spiral 2-dimensional NTI arrangement, the total distance between the center or first position in a packet stream is linearally closest to the sequentially ordered NTI blocks that come next, relative to all the other packet arrangements. We hypothesize that this property of the center spiral will make it significantly better than the other arrangements.

\subsection{Edge Spiral NTI Arrangement}

The edge spiral NTI arrangement is identical to the center spiral in shape and inspiration. However, instead of filling the NTI from the center, the edge spiral begins its fill pattern from the edge and slowly spirals inwards until it reaches the center NTI block. The edge spiral is the inverse of the center spiral. For example, a $5 \times 5$ edge spiral NTI would be arranged as follows:

\begin{tabular}{|c|c|c|c|c|}
\hline$n i^{-}$ & $\overline{\mathrm{n} 2}$ & $\overline{\mathbf{n} 3}$ & $\overline{\mathrm{n} 4}$ & $\overrightarrow{\mathbf{n} 5}$ \\
\hline n164 & $\overline{\mathrm{n}} \overline{17}$ & $\overline{\mathrm{n}} \overline{\mathbf{1}} \overline{\mathrm{s}}$ & $\overrightarrow{\mathbf{n} 1 \vec{S}_{1}}$ & n6! \\
\hline n15 ? & n24 & n25 & n20! & n7 \\
\hline n14! & n23! & n22 & _n21 & $n 8$ ! \\
\hline n13 & n12 & n11 & - & _n \\
\hline
\end{tabular}

Figure 9:Edge Spiral NTI Arrangement for $5 \times 5$ NTI with vectors to show fill pattern 
Depending on whether the NTI is packet-relative or time-relative, the content of each block of the NTI will be filled differently and have substantially changed meaning.

\subsubsection{Packet-Relative Network Traffic Images}

The simplest adaptation of an NTI is that of the packet-relative network traffic image. A packet relative NTI is constructed by placing the packets, more specificallythe features of interest of a packet, that comprise a packet stream sequentially onto an NTI image plane arranged in the manners described previously.

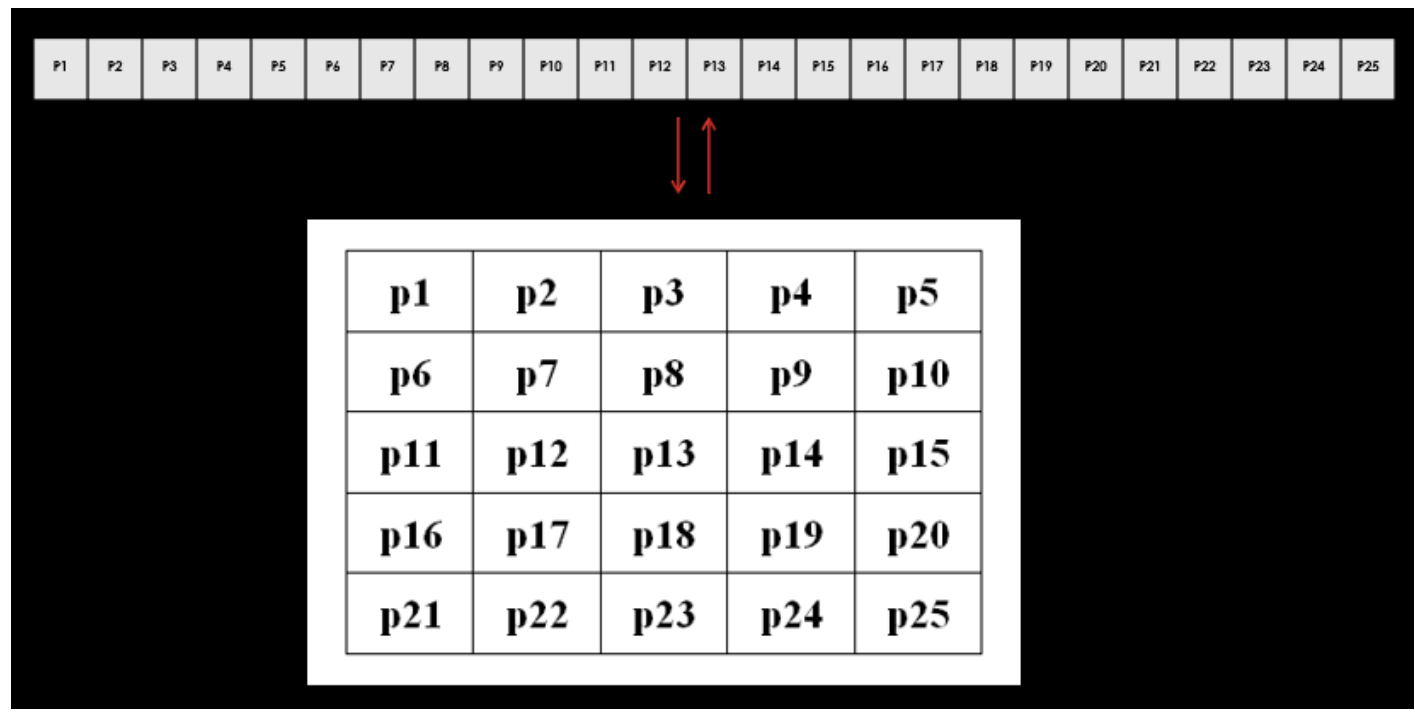

Figure 10: A simple packet-relative linear NTI mapping diagram.

The number of packets required to construct a packet-relative NTI is equal to the area of the NTI.

$$
P=N_{x} * N_{y}
$$

Equation 1:Formula for the Number of Packets Required to Construct a Packet-Relative NTI where P is the number of packets, Nx is the length dimension of the NTI and Ny is the width dimension of the NTI.

For example, a 17x17 NTI would require exactly 289 packets to construct. We generally construct odd dimension NTIs to avoid the need to zero-pad them when they 
are fed to convolutional neural network models, thus saving us a minor amount of computational complexity.

It's important to note that the number of unique sequential NTIs that can be generated from a packet stream is equivalent to the number of packets in a stream minus the number of packets required to construct the desired NTI.

$$
N_{p}=P_{s}-\left(N_{x} * N_{y}\right)
$$

Equation 2: Formula to calculate the total number of NTIs (Np) that can be generated from a given packet stream (Ps), where Ps is the number of packets captured for a given connection, Nx is length dimension of the NTI and Ny is the width dimension of the NTI.

For example, generating NTIs sized at $11 \times 15$ with a 1500 length packet stream can extract a total of 1500-(11*15), or 1335, unique NTIs. Ideally, a QoS engine could construct any one of these 1335 NTIs and classify the connection accurately. This is an important property to consider when building a QoS engine that is trying to maximize accuracy since it then becomes simple to feed multiple unique sequential NTIs and averaging the classification results - each extra NTI used to classify a connection only requires one extra packet than was generated in the previous NTI to generate it.

\subsubsection{Time-Relative Network Traffic Images}

A time-relative NTI separates a packet stream utilizing the time-dimension to determine what goes in each block of an NTI.

Time-relative NTIs are similar to packet-relative NTIs in that they follow the same NTI arrangement patterns as aforementioned in 3.2.2.1, however, each block of the NTI refers to a unique time-range offset from the initial reference time of the NTI. 


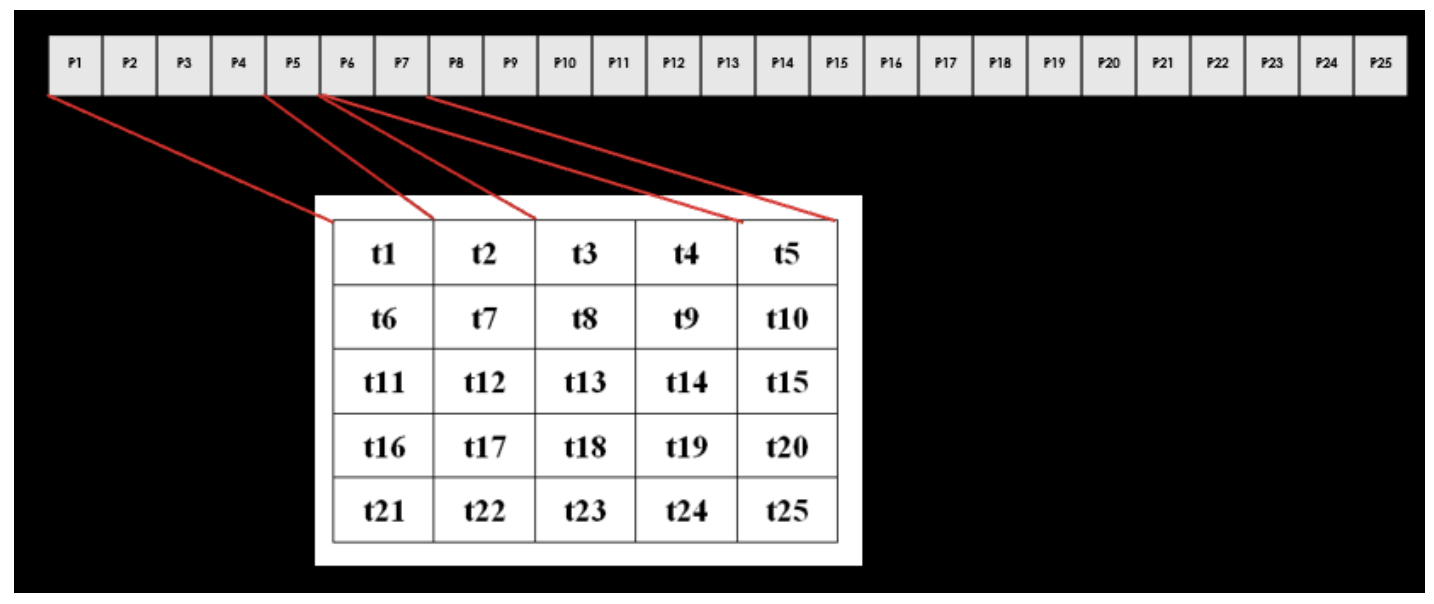

Figure 11: Packet Stream to linear Time-Relative NTI mapping example diagram

To construct a time-relative NTI from a packet flow, 4 parameters are required:

the intended $\mathrm{x}$ dimension of the NTI array $\left(\mathrm{N}_{\mathrm{x}}\right)$, the $\mathrm{y}$ dimension $\left(\mathrm{N}_{\mathrm{y}}\right)$, the NTI start time $\left(\mathrm{N}_{\mathrm{s}}\right)$ and the NTI duration $\left(\mathrm{N}_{\mathrm{d}}\right)$. The time duration that each NTI block encompasses $\left(\mathrm{N}_{\mathrm{b}}\right)$ is equivalent to the area of the NTI array divided by the NTI duration.

$$
N_{b}=\frac{N_{x} * N_{y}}{N_{d}}
$$

Equation 3: Formula to calculate the total duration of each time-block in a time-relative NTI where $N_{b}$ is the block duration, $N_{x}$ is the length dimension of the NTI, $N_{y}$ is the width dimension, and $N_{d}$ is the specified duration of the NTI

Unlike packet-relative NTIs, the number of packets required to generate a time-relative

NTI is completely arbitrary. However, in the event that a time-relative NTI spans over an idle period of a packet flow, experiments show that the addition of a fill threshold is necessary to determine whether a generated time-relative NTI is a valid NTI for traffic classification purposes.

If multiple packets fall within a single time range, the average of each packet feature, in our case the average packet header length, of each packet is computed to fill that time-block of the NTI. Depending on the parameters used to construct a time-relative 
NTI and the packet stream frequency, a time-relative NTI will often act as a packet frequency map for the connection stream.

Time-relative NTIs are lossy, meaning that information is lost when transforming from the 1-D packet flow to the 2-D NTI. It is inaccurate to transform a 2-D time relative NTI back to a packet sub-flow.

\subsubsection{Feature Selection}

So far, we have explained how we uniformly generate network traffic images that are the input of our machine learning models, however, we have yet to mention what features of the packet are actually used in each NTI block. A machine learning model doesn't understand how to interpret packet statistics natively. Given the TCPdump [33] packet statistic output that make up our datasets that we have already preprocessed accordingly to separate out only the packet flows of interest, each individual packet has quite a few number of features that can be derived.

2018-11-27 17:28:44.355167 IP (tos 0x0, tt7 56, id 64204, offset 0, flags [none], proto TCP (6), 1ength 40) 192.16.31.139.https > Shadowscout. shadowdomain.61901: Flags [F.], cksum 0xa114 (correct), seq 7227, ack 922, win 62, 1ength 0

Figure 12: TCPDump of single packet

Looking at the TCPDump [33] output in figure 11, we can easily derive atleast the following features: protocol, packet received time, source port, destination port, packet sequence number, which IP flags are set, the total IP packet length, application payload length, the protocol, the packet acknowledgement number and more. Beyond that, if we start to extract information from multiple packets instead of just a single packet, we can even look at such features as packet arrival deltas, the time difference between the current and last received packet, and packet round trip times to name a few. Even though using multiple features would likely increase classification accuracy, due to various constraints, 
this research will only utilize a 1-D feature vector for each NTI block--equivalent to a grayscale image in the image processing world.

Based on the research in [12] where Moore et al. exhaustively determine that the best twelve features for traffic classification given a packet flow, elaborated on in Chapter 2 above, we can derive that, since most of those features are related to packet size statistics, our NTIs will be well suited for traffic classification when fed the IP packet header length of each packet. Since we suspect that syn, ack and other header-only packets will be meaningful for traffic classification, we use the IP header length, which includes the application payload length, instead of just the application body length. Further feature based experimentation and multi-dimensional NTIs are reserved as the subject of future works.

\subsection{Machine Learning Model}

The concept of network traffic images is completely novel and has never before been exercised in previous research efforts. On the flip side of that, machine learning, specifically convolutional neural networks $(\mathrm{CNN})$, have been around for years now and have shown tremendous results when both properly designed and trained. Model competitions and years of experimentation have proven the VGG-16 CNN model to be one of the best in its class for image recognition. [16] The primary goal of this research is to evaluate the usability of network traffic images for the purposes of network traffic classification, not to develop new machine learning models. As such, we base our primary machine learning model off of the VGG-16 CNN presented in [16] with just a few tiny variations to make learning faster. We attempt to use the same model for each of 
our experiments so that it is as minimal a variable as possible in evaluating NTIs. Our base model is structured as follows: 


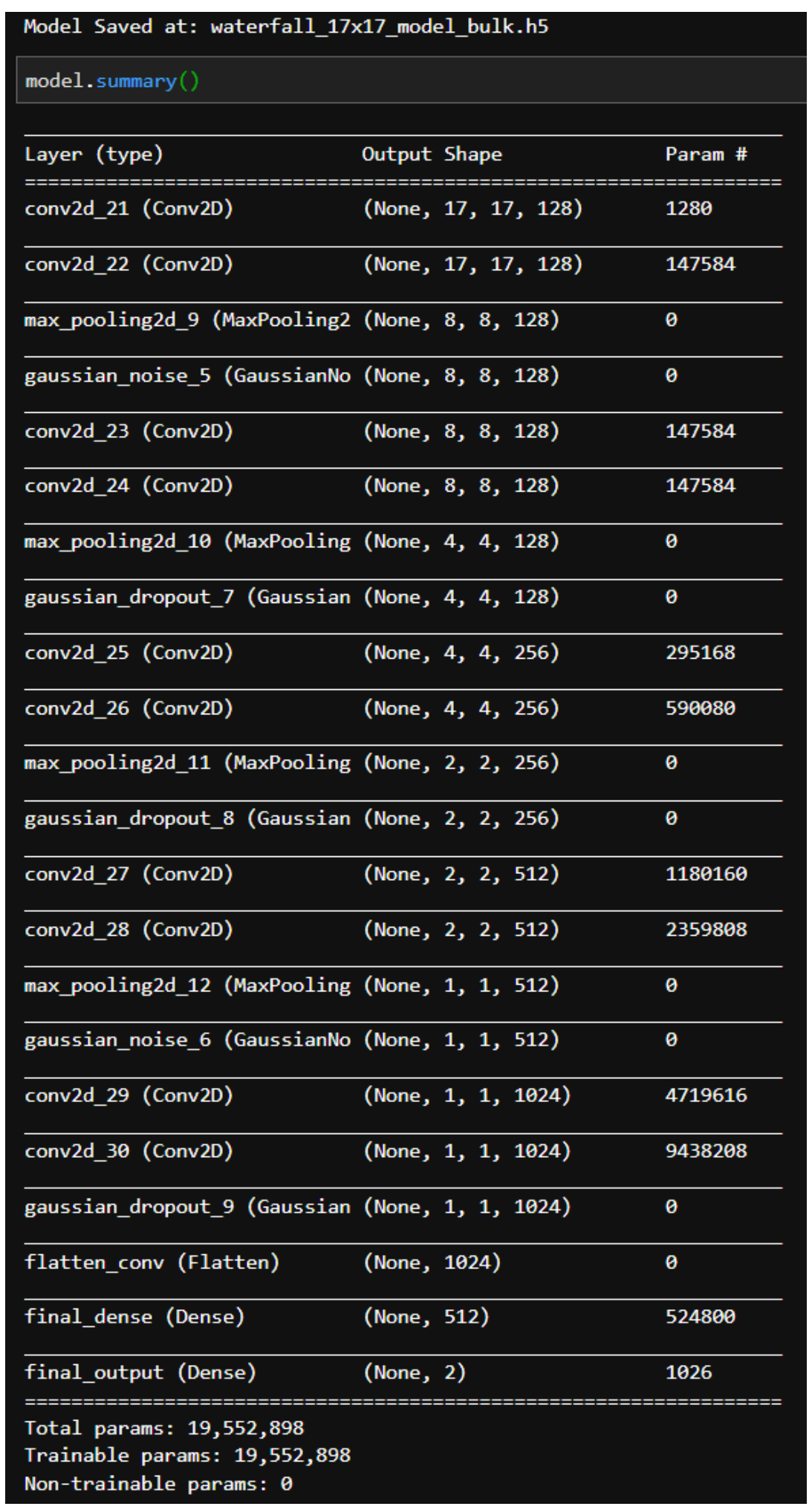

Figure 13: VGG-16 Model Variant Used as Starting Point for all NTI Experiments. 
Since the size of our smallest tested NTIs is $17 x 17$, too small to work with all the pooling structured in the original model in [16], we vary the VGG-16 model to make it usable by removing one max-pooling layer and adding some dropout. In addition to using a slight variant of the VGG-16 model, to help minimize the human controlled variations required for fine-tuning our models and ultimately spend less computational time training our models, we utilized an adadelta optimizer that automatically adjusts the model learning rate. [35] The adadelta optimizer allows us to train our model without manually setting a learning rate, is insensitive to other hyper-parameters and robust to varying architecture choices. [35]

The only other additions made to the machine learning process to ensure more efficient and easier training cycles is that callbacks were added to stop training early given certain conditions. Two training conditions in particular were monitored: training loss and validation accuracy. When the validation accuracy of our models hit $95 \%$, we stopped training the model and used that as our final predictor for the test data. Also, when training loss fell below 0.23 , we also immediately stopped training the model as any further training would cause overfitting and only ever reduce model accuracy.

Creating the perfect machine learning model for any given task is proven to be NP-Complete in [36]. No doubt, certain model variations exist from what was selected, trained and tested in this research that would improve the accuracy of certain NTIs. Such experimentation is beyond the scope of this research and reserved for future works.

\subsection{Experiments}

There are two primary classes of experiments that will be executed per this research, packet-relative NTI experiments and time-relative NTI experiments. For each 
experiment type, we will be testing all five previously described NTI arrangements:

Linear, Diagonal, Waterfall, Center Spiral and Edge Spiral. For each NTI arrangement, we will be testing two different sized NTIs, 17x17 NTIs and 49x49 NTIs. For each NTI size, we will be testing at each of the three different levels of classification, bulk vs all, QoS based and class based.

For each experiment, two tests are run to evaluate the effectiveness of the relative NTI for that classification test. First we do a normal test prediction, the dataset of which includes completely unique packet flows from applications that were used to train the machine learning model. The second, more difficult test is conducted with zero-day application data. That is, applications from the same classes that we categorized but that have never had any training data fed to the machine learning model. Each experiment will reveal different facts about the effectiveness of NTIs for class-based network traffic classification.

\subsubsection{Packet-Relative NTI Experiments}

A mindmap detailing all of the packet-relative NTI experiments planned to be executed can be seen below. 


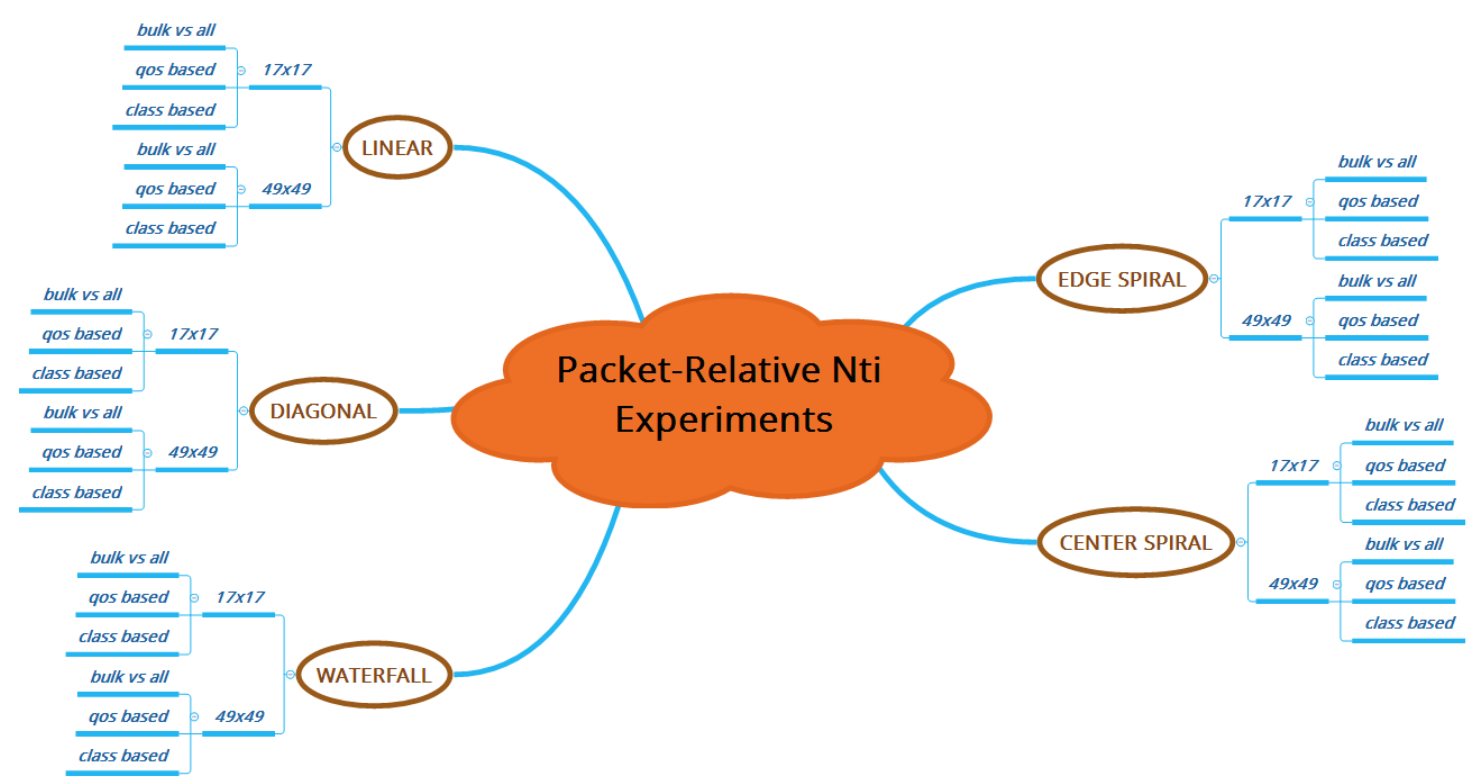

Figure 14: Mindmap detailing all Packet-Relative NTI Experiments to be executed

In order for a connection stream to be classifiable by models using these NTIs, they must, at minimum, have transferred 289 packets in a single packet stream for the $17 \times 17$ experiments. For the 49x49 experiments, they must, at minimum, have transferred 2401 packets during the lifetime of a single packet stream. Just to reiterate, NTI based methods are not intended for short-lived connection streams, for example, those that are commonly found in simple DNS calls or when using RESTful queries. This methodology is exclusively for long-duration connections. Of all the data captured for this research, around $99 \%$ of connections were not applicable to be classified. On the corollary, however, the $1 \%$ of connections that were classified were responsible for utilizing $99 \%$ of the bandwidth captured.

Each 17x17 packet-relative experiment has 624,608 unique NTIs. 120,000 NTIs from unique connection streams are reserved for testing our results and never fed to the machine learning model. 87,593 unique NTIs captured from applications that never had 
any data fed to the machine learning models are reserved for zero-day testing results.

417,015 unique NTIs are used to train and validate our model with a $12 \%$ train-validate split.

Each 49x49 packet-relative experiment has 202,881 unique NTIs. There were many more NTIs generated, however, we were unable to fit them all into memory for training purposes (our training machine had 36GB of RAM total) so we had to reduce the training sets. 52,008 unique NTIs from connection streams that never had any NTIs fed into our model were reserved for testing purposes. 46,857 unique NTIs from applications that never had any NTIs trained with our model were reserved for zero-day testing results. 104,016 unique NTIs were reserved for training and validation purposes with a $12 \%$ train-validate split.

\subsubsection{Time-Relative NTI Experiments}

A mindmap detailing all of the time-relative NTI experiments planned to be executed can be seen below. 

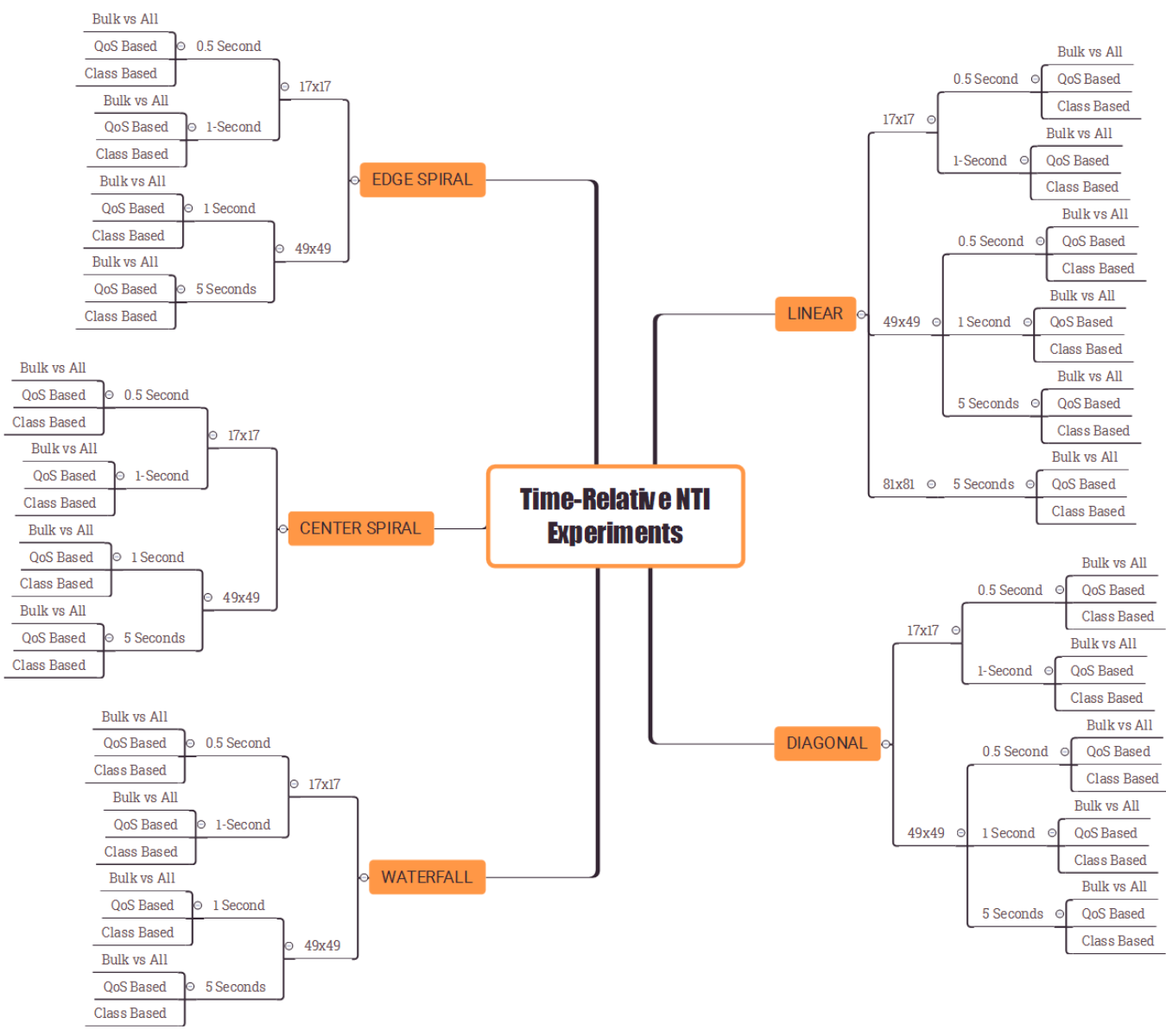

Experiments
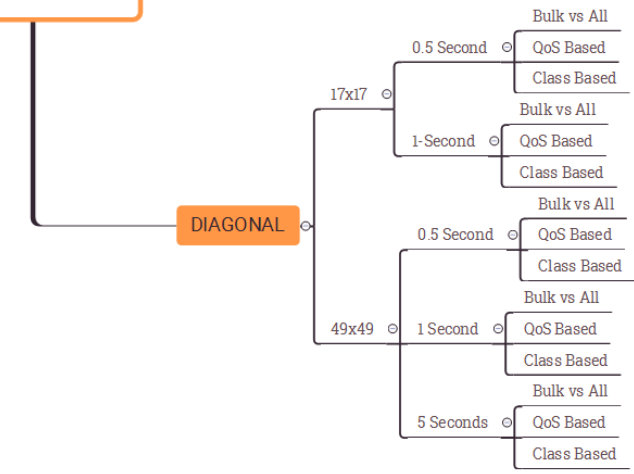

Figure 15:Mindmap detailing all Time-Relative NTI Experiments to be executed.

For time-relative NTIs, in addition to the packet arrangement and NTI size

parameters that must be set to generate the NTIs, you must also include a global time parameter to control how big of a time range each NTI should encompass. Our tests were planned with 0.5 second, 1 second, and 5 second intervals, however, that would have to change for reasons described in the results section of chapter 4 . Given the changes detailed in chapter 4 , further description of our originally planned time-relative experiments will be avoided here. 


\section{CHAPTER 4 - RESULTS}

\subsection{Packet-Relative NTI Experiment Results}

The results of both the $17 \times 17$ and 49x49 packet-relative NTI experiment sets reveal alot about the positives and shortcomings of the approach to traffic classification described in this research.

\subsubsection{7x17 Packet-Relative NTI Results}

The test results for the 17x17 packet-relative NTI experiments were as follows:

Table 2:17x17 packet-relative NTI experimentation results

\begin{tabular}{|c|c|c|c|c|}
\hline Class Type & NTI Arrangement & NTI Size & Test Result & Test 0-Day Result \\
\hline Bulk vs All & Linear & $17 \times 17$ & $97.535 \%$ & $58.791 \%$ \\
\hline QoS & Linear & $17 \times 17$ & $97.192 \%$ & $35.567 \%$ \\
\hline App Classes & Linear & $17 \times 17$ & $95.411 \%$ & $35.788 \%$ \\
\hline Bulk vs All & Diagonal & $17 \times 17$ & $83.495 \%$ & $49.68 \%$ \\
\hline QoS & Diagonal & $17 \times 17$ & $75.270 \%$ & $36.821 \%$ \\
\hline App Classes & Diagonal & $17 \times 17$ & $73.680 \%$ & $36.446 \%$ \\
\hline Bulk vs All & Waterfall & $17 \times 17$ & $85.441 \%$ & $47.310 \%$ \\
\hline QoS & Waterfall & $17 \times 17$ & $94.715 \%$ & $55.847 \%$ \\
\hline App Classes & Waterfall & $17 \times 17$ & $91.408 \%$ & $57.821 \%$ \\
\hline Bulk vs All & Center Spiral & $17 \times 17$ & $97.808 \%$ & $\mathbf{5 8 . 5 3 7 \%}$ \\
\hline QoS & Center Spiral & $17 \times 17$ & $95.467 \%$ & $34.412 \%$ \\
\hline App Classes & Center Spiral & $17 \times 17$ & $89.237 \%$ & $32.953 \%$ \\
\hline Bulk vs All & Edge Spiral & $17 \times 17$ & $83.216 \%$ & $48.282 \%$ \\
\hline QoS & Edge Spiral & $17 \times 17$ & $84.170 \%$ & $50.117 \%$ \\
\hline App Classes & Edge Spiral & $17 \times 17$ & $80.628 \%$ & $59.159 \%$ \\
\hline
\end{tabular}

Comparing the packet arrangement factor of these experiments, we can clearly

derive that packet arrangement does have a significant effect on classification accuracy depending on the classification type being used. In the most extreme case, we can see an 
accuracy degradation of $14 \%$ between the best and worst performing Bulk vs non-bulk classifiers.

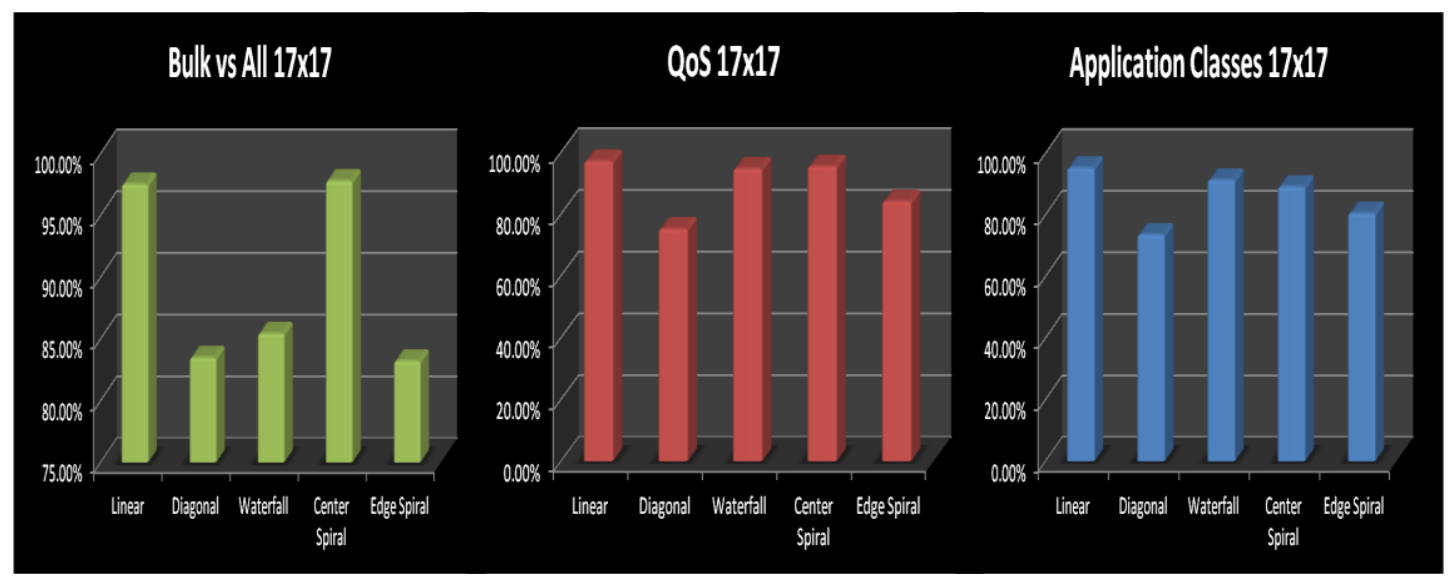

Figure 16: $17 x 17$ NTI Test Result Charts Plotted with Respect to Packet Arrangement

These results indicate that the best packet arrangement for a simple Bulk vs All classifier is the center_spiral arrangement, though only by a hair over the linear arrangement. The best NTI arrangement for both the QoS identifier application class based identifiers is a linear arrangement. This was an unexpected outcome for these experiments. Overall, we can achieve over $95 \%$ accuracy with all classifier types for known, trained-for applications. However, when it comes to zero-day application testing, the best accuracy found for the application classes classifier is $59 \%$ with the edge spiral arrangement. Though better than the other arrangements, it is still very poor results overall for zero-day application testing. The implications of this are discussed in the conclusion.

\subsubsection{9x49 Packet-Relative NTI Results}

When attempting to train the base VGG-16 model, as described in Chapter 3, with 49x49 input NTIs, we ran into the issue where the model could simply never converge 
and fit to the training data. After leaving the experiment running for nearly 16 hours, I decided that the model simply wasn't complex enough to be able to handle the complexity of the data features present in the 49x49 NTI and abandoned the experiment. To augment the model slightly but still retain most of the original shape of the VGG-16, I simply added two dense 512 layers after the CNN. This enabled the model to finally be able to fit to the training data after some number of epochs. I was finally able to get results, however, due to the model modification, they are not entirely comparable to those obtained with the $17 \times 17$ experiments.

The results for the 49x49 packet-relative NTI experiments are as follows:

Table 3: 49x49 packet-relative NTI experimentation results

\begin{tabular}{ccccc}
\hline Class Type & NTI Arrangement & NTI Size & Test Result & Test 0-Day Result \\
\hline Bulk vs All & Linear & $49 \times 49$ & $\mathbf{8 5 . 8 4 8 \%}$ & $\mathbf{4 4 . 7 8 9 \%}$ \\
\hline QoS & Linear & $49 \times 49$ & $80.859 \%$ & $48.292 \%$ \\
\hline App Classes & Linear & $49 \times 49$ & $\mathbf{6 4 . 3 4 9 \%}$ & $\mathbf{4 4 . 2 3 0 \%}$ \\
\hline Qulk vs All & Diagonal & $49 \times 49$ & $95.656 \%$ & $58.573 \%$ \\
\hline App Classes & Diagonal & $49 \times 49$ & $77.326 \%$ & $\mathbf{2 8 . 1 2 9 \%}$ \\
\hline Bulk vs All & Wiagonal & $49 \times 49$ & $63.664 \%$ & $26.757 \%$ \\
\hline QoS & Waterfall & $49 \times 49$ & $95.078 \%$ & $\mathbf{7 0 . 4 8 3 \%}$ \\
\hline App Classes & Waterfall & $49 \times 49$ & $\mathbf{6 5 . 4 3 1 \%}$ & $\mathbf{4 4 . 2 6 0 \%}$ \\
\hline Bulk vs All & Center Spiral & $49 \times 49$ & $96.392 \%$ & $56.660 \%$ \\
\hline QoS & Center Spiral & $49 \times 49$ & $\mathbf{8 3 . 3 3 3 \%}$ & $\mathbf{2 6 . 9 0 7 \%}$ \\
\hline App Classes & Center Spiral & $49 \times 49$ & $59.669 \%$ & $27.897 \%$ \\
\hline Bulk vs All & Edge Spiral & $49 \times 49$ & $\mathbf{7 8 . 7 6 7 \%}$ & $\mathbf{7 0 . 9 5 2 \%}$ \\
\hline QpoS Classes & Edge Spiral & $49 \times 49$ & $75.031 \%$ & $\mathbf{6 4 . 0 8 0 \%}$ \\
\hline Despiral & $49 \times 49$ & $\mathbf{7 2 . 3 8 1 \%}$ & $\mathbf{6 4 . 3 8 1 \%}$ \\
\hline
\end{tabular}

Despite utilizing more packets to fill up the $49 \times 49$ NTI, it seems that the $17 x 17$

NTIs perform better in general. 


\subsection{Time-Relative NTI Experiment Results}

The time-relative NTI experiments were at first inconclusive due to an original design issue. After redesigning them with new specifications, we are able to clearly see their effectiveness for internet traffic classification.

\subsubsection{Time-Relative NTI Experiment Design Issue}

After generating the time relative NTIs as described in chapter 3 and briefly evaluating them with the VGG-16 based machine learning model, I quickly noticed that the classification results being outputted were absolutely terrible. After further investigation into the NTIs themselves, I learned that a huge number of the generated time-relative NTIs were almost completely empty for certain application types. For example, here are the packet values for one of the NTIs generated from a CSGO capture in the games class using a 17x17 1-second time-relative NTI: 


\begin{tabular}{|c|c|c|c|c|c|c|c|c|c|c|c|c|c|c|c|c|c|c|}
\hline 1 & 0 & & 0 & 0 & 0 & 0 & 0 & 0 & 0 & 0 & 0 & 0 & 0 & 0 & 0 & 0 & 0 & \\
\hline 2 & 0 & 0 & 0 & 0 & 0 & 0 & 0 & 0 & 0 & 0 & 0 & 0 & 0 & 0 & 0 & 0 & 0 & \\
\hline 3 & 0 & 0 & 0 & 0 & 0 & 0 & 0 & 0 & 0 & 0 & 0 & 0 & 0 & 0 & 0 & 0 & 0 & \\
\hline 4 & 0 & 0 & 0 & 0 & 0 & 0 & 0 & 0 & 0 & 0 & 0 & 0 & 0 & 0 & 0 & 0 & 0 & \\
\hline 5 & 0 & 0 & 0 & 0 & 0 & 0 & 0 & 0 & 0 & 0 & 0 & 0 & 0 & 0 & 0 & 0 & 0 & \\
\hline 6 & 0 & 0 & 0 & 0 & 0 & 0 & 0 & 0 & 0 & 0 & 0 & 0 & 0 & 0 & 0 & 0 & 0 & \\
\hline 7 & 0 & 0 & 0 & 0 & 0 & 0 & 0 & 0 & 0 & 0 & 0 & 0 & 0 & 0 & 0 & 0 & 0 & \\
\hline 8 & 0 & 0 & 0 & 0 & 0 & 0 & 0 & 0 & 0 & 0 & 0 & 0 & 0 & 0 & 0 & 0 & 0 & \\
\hline 9 & 0 & 0 & 0 & 0 & 0 & 0 & 0 & & 58 & 0 & 0 & 0 & 0 & 0 & 0 & 0 & 0 & 0 \\
\hline 10 & 0 & 0 & 0 & 0 & 0 & 0 & 0 & 0 & 0 & 0 & 0 & 0 & 0 & 0 & 0 & 0 & 0 & \\
\hline 11 & 0 & 0 & 0 & 0 & 0 & 0 & 0 & 0 & 0 & 0 & 0 & 0 & 0 & 0 & 0 & 0 & 0 & \\
\hline 12 & 0 & 0 & 0 & 0 & 0 & 0 & 0 & 0 & 0 & 0 & 0 & 0 & 0 & 0 & 0 & 0 & 0 & \\
\hline 13 & 0 & 0 & 0 & 0 & 0 & 0 & 0 & 0 & 0 & 0 & 0 & 0 & 0 & 0 & 25 & 8 & 0 & 0 \\
\hline 14 & 0 & 0 & 0 & 0 & 0 & 0 & 0 & 0 & 0 & 0 & 0 & 0 & 0 & 0 & 0 & 0 & 0 & \\
\hline 15 & 0 & 0 & 0 & 0 & 0 & 0 & 0 & 0 & 0 & 0 & 0 & 0 & 0 & 44 & 14 & 0 & 0 & 0 \\
\hline 16 & 0 & 0 & 0 & 0 & 0 & 0 & 0 & 0 & 0 & 0 & 0 & 0 & 0 & 0 & 0 & 0 & 0 & \\
\hline 17 & 0 & 0 & 0 & 0 & 0 & 0 & 0 & 0 & 0 & 0 & 0 & 0 & 0 & 0 & 0 & 0 & 0 & \\
\hline
\end{tabular}

Figure 17: 17x17 CSGO Time-Relative NTI with 1\% fill

In the entire 289 block NTI shown in the figure above, only 3 blocks are populated with actual packets. The really low classification accuracy observed appeared to be because most of the NTIs were $95 \%$ or more empty. To work-around this problem, all time relative NTIs were reprocessed and any NTI with less than $20 \%$ fill was discarded. However, this reprocessing resulted in the deletion of almost all of the NTIs from the streams and games application categories, thus nullifying the feasibility of the experiment. With the 49x49 time relative NTIs, over $85 \%$ of all NTIs were deleted.

It soon became clear that the time-relative experiments would have to be redesigned to encompass packet densities that games and streaming applications exhibited. With the previous $17 \times 171$ second time relative NTI, each NTI block encompassed a total time range of $3.46 \mathrm{~ms}$. With the 49x49 5 second time relative NTI, each NTI block encompassed a range of $2.08 \mathrm{~ms}$. These block ranges were simply too 
short for application types that steadily sent small numbers of packets over long durations of time. Two new experiment sets were then planned with time-relative NTIs. The first redesigned experiment set uses $17 \times 17-5$ second NTIs with a 10 percent fill threshold, allowing for $17.3 \mathrm{~ms}$ time ranges for each block of the NTI. Instead of using 49x49 blocks which seemed large for this after preliminary experiment results, the second redesigned experiment set uses a $25 \times 25-10$ second NTI with a 5 percent fill threshold. Similarly, this enables a $16 \mathrm{~ms}$ time range for each block of the NTI.

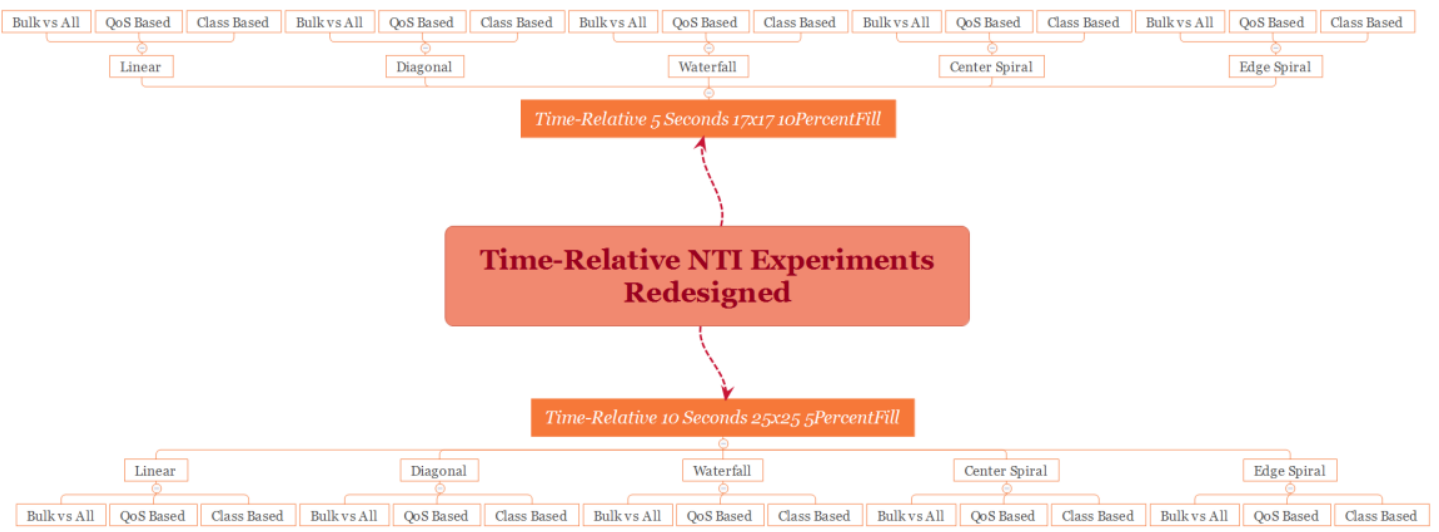

Figure 18: Mindmap showing the redesign of the Time-Relative NTI Experiments with more appropriate time ranges

Regenerating the NTIs with the redesigned experiment specifications resulted in 484,828 usable NTIs for the $25 \times 25$ experiment set and 378,905 usable NTIs for the $17 \times 17$ experiment set. Note, the $25 \times 25$ experiments have more usable NTIs generated from the same packet stream than the $17 \times 17$ experiments due to the different packet fill requirements placed: 10 percent fill versus 5 percent fill, the thresholds used to consider each generated NTI a usable NTI. 


\subsubsection{7x17 5-second Time-Relative NTI with 10\% Fill Results}

After redesigning the time-relative NTI experiments and re-executing the entire experiment set, we are able to finally get $17 \times 17$-second time-relative NTIs with $10 \%$ fill threshold experimental results as follows:

\begin{tabular}{|c|c|c|c|c|c|c|}
\hline Class Type & $\begin{array}{c}\text { NTI } \\
\text { Arrangement }\end{array}$ & NTI Size & $\begin{array}{l}\text { NTI Time } \\
\text { Span }\end{array}$ & $\begin{array}{l}\text { NTI Fill } \\
\text { Threshold }\end{array}$ & $\begin{array}{c}\text { Test } \\
\text { Result }\end{array}$ & $\begin{array}{c}\text { Test 0-Day } \\
\text { Result }\end{array}$ \\
\hline $\begin{array}{c}\text { Bulk vs } \\
\text { All }\end{array}$ & Linear & $17 \times 17$ & $5 \mathrm{~s}$ & $10 \%$ & $95.997 \%$ & $95.341 \%$ \\
\hline QoS & Linear & $17 \times 17$ & $5 \mathrm{~s}$ & $10 \%$ & $95.149 \%$ & $95.858 \%$ \\
\hline $\begin{array}{c}\text { App } \\
\text { Classes }\end{array}$ & Linear & $17 \times 17$ & $5 s$ & $10 \%$ & $93.278 \%$ & $65.443 \%$ \\
\hline $\begin{array}{c}\text { Bulk vs } \\
\text { All }\end{array}$ & Diagonal & $17 \times 17$ & $5 \mathrm{~s}$ & $10 \%$ & $95.977 \%$ & $96.506 \%$ \\
\hline QoS & Diagonal & $17 \times 17$ & $5 s$ & $10 \%$ & $96.691 \%$ & $96.312 \%$ \\
\hline $\begin{array}{c}\text { App } \\
\text { Classes }\end{array}$ & Diagonal & $17 \times 17$ & $5 \mathrm{~s}$ & $10 \%$ & $91.561 \%$ & $50.800 \%$ \\
\hline $\begin{array}{c}\text { Bulk vs } \\
\text { All }\end{array}$ & Waterfall & $17 \times 17$ & $5 s$ & $10 \%$ & $95.779 \%$ & $96.162 \%$ \\
\hline QoS & Waterfall & $17 \times 17$ & $5 \mathrm{~s}$ & $10 \%$ & $95.432 \%$ & $95.640 \%$ \\
\hline $\begin{array}{c}\text { App } \\
\text { Classes }\end{array}$ & Waterfall & $17 \times 17$ & $5 s$ & $10 \%$ & $95.241 \%$ & $41.176 \%$ \\
\hline $\begin{array}{c}\text { Bulk vs } \\
\text { All }\end{array}$ & $\begin{array}{l}\text { Center } \\
\text { Spiral }\end{array}$ & $17 \times 17$ & $5 \mathrm{~s}$ & $10 \%$ & $98.241 \%$ & $96.315 \%$ \\
\hline QoS & $\begin{array}{l}\text { Center } \\
\text { Spiral }\end{array}$ & $17 \times 17$ & $5 \mathrm{~s}$ & $10 \%$ & $98.584 \%$ & $95.597 \%$ \\
\hline $\begin{array}{c}\text { App } \\
\text { Classes }\end{array}$ & $\begin{array}{l}\text { Center } \\
\text { Spiral }\end{array}$ & $17 \times 17$ & $5 \mathrm{~s}$ & $10 \%$ & $\mathbf{8 2 . 5 7 0 \%}$ & $38.357 \%$ \\
\hline $\begin{array}{c}\text { Bulk vs } \\
\text { All }\end{array}$ & Edge Spiral & $17 \times 17$ & $5 s$ & $10 \%$ & $98.467 \%$ & $96.633 \%$ \\
\hline QoS & Edge Spiral & $17 \times 17$ & $5 \mathrm{~s}$ & $10 \%$ & $98.283 \%$ & $96.437 \%$ \\
\hline $\begin{array}{c}\text { App } \\
\text { Classes }\end{array}$ & Edge Spiral & $17 \times 17$ & $5 \mathrm{~s}$ & $10 \%$ & $83.187 \%$ & $34.150 \%$ \\
\hline
\end{tabular}

Table 4: 17x17 5-second Time relative NTI with forced $10 \%$ fill results

The results from the $17 \times 175$-second time relative NTIs are seemingly the most promising of all the varying NTI applications for the purposes of class-based 
classification. These time-relative NTIs are the only ones with seemingly acceptable accuracy levels for predicting the class of 0-day data.

\subsubsection{5x25 10-second Time-Relative NTI with 5\% Fill Threshold Results}

Similar to the $49 \times 49$ packet-relative NTIs previously mentioned, the $25 \times 25$ timerelative NTI experiments occasionally encountered the issue where the stock VGG-16 was unable to converge on the training set. With this situation, however, I only ever needed to add a single dense 512 layer to give the model enough complexity to handle the features of the $25 \times 25$ sized NTIs. The test results for the $25 \times 2510$-second time-relative NTI experiments with a forced 5\% fill threshold were as follows:

Table 5: 25x25 10-second Time relative NTI with forced 5\% fill results

\begin{tabular}{|c|c|c|c|c|c|c|}
\hline $\begin{array}{l}\text { Class } \\
\text { Type }\end{array}$ & $\begin{array}{c}\text { NTI } \\
\text { Arrangement }\end{array}$ & NTI Size & $\begin{array}{l}\text { NTI } \\
\text { Time } \\
\text { Span }\end{array}$ & $\begin{array}{l}\text { NTI Fill } \\
\text { Threshold }\end{array}$ & $\begin{array}{c}\text { Test } \\
\text { Result }\end{array}$ & $\begin{array}{c}\text { Test 0-Day } \\
\text { Result }\end{array}$ \\
\hline $\begin{array}{c}\text { Bullk vs } \\
\text { All }\end{array}$ & Linear & $25 \times 25$ & $10 \mathrm{~s}$ & $5 \%$ & $94.426 \%$ & $73.306 \%$ \\
\hline QoS & Linear & $25 \times 25$ & $10 \mathrm{~s}$ & $5 \%$ & $98.611 \%$ & $73.440 \%$ \\
\hline $\begin{array}{c}\text { App } \\
\text { Classes }\end{array}$ & Linear & $25 \times 25$ & 10s & $5 \%$ & $97.160 \%$ & $54.256 \%$ \\
\hline $\begin{array}{c}\text { Bullk vs } \\
\text { All }\end{array}$ & Diagonal & $25 \times 25$ & $10 \mathrm{~s}$ & $5 \%$ & $98.215 \%$ & $74.518 \%$ \\
\hline QoS & Diagonal & $25 \times 25$ & $10 \mathrm{~s}$ & $5 \%$ & $97.895 \%$ & $73.829 \%$ \\
\hline $\begin{array}{c}\text { App } \\
\text { Classes }\end{array}$ & Diagonal & $25 \times 25$ & $10 \mathrm{~s}$ & $5 \%$ & $93.288 \%$ & $41.741 \%$ \\
\hline $\begin{array}{c}\text { Bulk vs } \\
\text { All }\end{array}$ & Waterfall & $25 \times 25$ & $10 \mathrm{~s}$ & $5 \%$ & $96.637 \%$ & $66.910 \%$ \\
\hline QoS & Waterfall & $25 \times 25$ & $10 \mathrm{~s}$ & $5 \%$ & $94.286 \%$ & $71.341 \%$ \\
\hline $\begin{array}{c}\text { App } \\
\text { Classes }\end{array}$ & Waterfall & $25 \times 25$ & $10 \mathrm{~s}$ & $5 \%$ & $90.492 \%$ & $23.384 \%$ \\
\hline $\begin{array}{c}\text { Bulk vs } \\
\text { All }\end{array}$ & $\begin{array}{l}\text { Center } \\
\text { Spiral }\end{array}$ & $25 \times 25$ & $10 \mathrm{~s}$ & $5 \%$ & $97.816 \%$ & $78.983 \%$ \\
\hline QoS & $\begin{array}{l}\text { Center } \\
\text { Spiral }\end{array}$ & $25 \times 25$ & $10 \mathrm{~s}$ & $5 \%$ & $98.540 \%$ & $75.424 \%$ \\
\hline
\end{tabular}




\begin{tabular}{ccccccc}
$\begin{array}{c}\text { App } \\
\text { Classes }\end{array}$ & $\begin{array}{c}\text { Center } \\
\text { Spiral }\end{array}$ & $25 \times 25$ & 10 s & $5 \%$ & $97.470 \%$ & $\mathbf{3 7 . 6 6 9 \%}$ \\
$\begin{array}{c}\text { Bulk vs } \\
\text { All }\end{array}$ & Edge Spiral & $25 \times 25$ & 10 s & $5 \%$ & $98.927 \%$ & $\mathbf{7 6 . 5 0 7 \%}$ \\
QoS & Edge Spiral & $25 \times 25$ & 10 s & $5 \%$ & $97.999 \%$ & $\mathbf{7 5 . 8 3 2 \%}$ \\
$\begin{array}{c}\text { App } \\
\text { Classes }\end{array}$ & Edge Spiral & $25 \times 25$ & 10 s & $5 \%$ & $91.350 \%$ & $\mathbf{3 6 . 7 1 1 \%}$ \\
\hline
\end{tabular}

Unlike the previous NTI experiment types that showed significant variation in the test accuracy due to the packet arrangement, the $25 \times 25$ time-relative NTI never had less than $90 \%$ test accuracy for known application types. That's quite promising and indicates that it was able to fit to the training data really well. 


\section{CHAPTER 5 - CONCLUSION}

\subsection{Lessons Learned}

All of the highest test accuracies presented in this paper were the result of the time-relative NTIs. The edge spiral 25×25-10 second time relative NTI had the highest accuracy at $98.93 \%$ accuracy. The linear $25 \times 25-10$ second time relative NTIs produced the highest QoS classifier accuracy at $98.61 \%$. The center spiral 25x25-10 second time relative NTIs produced the highest application class classifier accuracy at $97.47 \%$. However, these same models, which were the most accurate on the test data, a collection of unique sessions from applications the models were trained with, performed just slightly above average with regards to the 0-day test data. I believe that the lesson here is in of the most important realized in this research. It is very human to look at what two different applications are doing and classify them as belonging to a group and claim that they communicate similarly. To a computer, however, traffic that we might group together as being most similar may, in fact, be very different with regards to the actual packet transmission patterns involved.

The packet arrangement question investigated by our experimentation ultimately seems inconclusive. Under different circumstances, different arrangements outperform each other. The differences, in some of the cases, are seemingly negligible while in other cases, quite significant. There doesn't appear to be a pattern that can be derived from it. For example, with the $17 \times 17$ time relative NTIs, the edge spiral pattern sometimes performs really well, but then in another experiment where you would expect it to shine also, it suddenly performs the worst of all the packet arrangement patterns. There is no 
clear best packet arrangement for generating an NTI that can be determined from these experiments. They each shine under different circumstances with varying models.

Our high test accuracy indicates that network traffic images are definitely viable for internet traffic classification.

\subsection{Future Works}

- The focus of this research has been exclusively on using network traffic images and convolutional neural networks on class of service based traffic classification intended for use in a QoS engine. Given the near perfect accuracy of the class based experiments with multiple variants of the $17 \times 17$ NTIs, the possibility of using network traffic images with deep neural networks should be investigated as a potential replacement to traditional DPI techniques.

- When it was discovered that the VGG-16 based model was unable to converge on the 49x49 packet-relative NTIs and certain 25x25 time-relative NTIs, ideally due to the large number of features within the NTIs, the decision was made to add dense layers with 512 nodes each as well as a small amount of dropout. This is exactly the kind of model experimentation that was attempted to be strictly avoided in this research. By shifting from the well known VGG-16 to our augmented VGG-16 with extra dense layers, this research changed from using a tried and true champion of a model [16] to something never before tested at scale. That is likely the reason why the results from the $49 \times 49$ and $25 \times 25$ NTIs were, in some instances, quite inferior to that of the simpler $17 \times 17$ NTIs. Increased model experimentation in the future on larger NTIs would likely yield superior results to the $17 \times 17$ NTIs. 
- Time-Relative NTIs sometimes have small holes, 0-value NTI blocks, between otherwise consistent feature patterns. In image manipulation, blurring techniques and interpolation have proven useful in increasing classification accuracy. There is no reason why such techniques couldn't be applied to NTIs and experimented with.

- Images in the real world are mostly 3-dimensional (given the RGB channel), however, the images that are composed of the NTIs defined here only have a 1dimension feature channel, namely the IP packet header length, for each NTI block. Further feature based experimentation and the utilization of multidimensional NTIs has the potential to significantly increase classification accuracy.

- Though a large number of unique connection streams and NTIs were used in this research, only 14 different applications were actually used in training the machine learning model. I would suspect that training our machine learning models with a wider variety of applications for each class would improve their ability to predict zero-day application traffic.

- The accuracy of the methodologies presented in this paper are high, however, no investigation has been done thus far as to whether the techniques used here are viable in high-performance demanding network hardware. Though we hypothesize that NTI based technology should work in the real world for traffic classification purposes since deep learning models work on mobile devices and DPI, which is naturally more computationally expensive since it inspects 
application layer data, is use on real internet routers, performance evaluations are an important next step for the methodology presented in this research.

\subsection{Final Remarks}

In this paper, we have looked at traditional approaches to network traffic classification, including port mapping and deep packet inspection techniques, and discussed the shortcomings with them in the modern world. We then looked at the two broad categories of research-level network traffic classification techniques, including host-behavior based techniques and packet flow statistic based techniques, and touched on the usefulness and short-comings of each approach along with the assumptions and requirements of each method. We then were introduced to the concept of network traffic images and the methodology by which the usefulness of this would be verified. We designed and analyzed the results of 60 unique experiments with 120 different accuracy measurements provided. The results prove that deep learning techniques combined with network traffic images can achieve the highest accuracy reported with regards to traffic classification for packet subflows to date. Though there is a great deal more to research with this methodology before it could ever be incorporated into real-world traffic classifiers, it has, thus far, shown tremendous promise. To the best of my knowledge, the research presented in this thesis is the first of its class in two significant ways: It is the

first to apply deep learning methodologies, specifically convolutional neural networks, to the field of internet traffic classification and, secondly, it is the first to introduce the concept of network traffic images as training and testing inputs for machine learning models. The results shown in this research are encouraging and the possibilities that can be explored in derivative works are seemingly endless. 


\section{REFERENCES}

[1] Pew Research Center, "pewinternet.org," Pew Research Center, 5 February 2018. [Online]. Available: http://www.pewinternet.org/fact-sheet/internet-broadband/.

[2] D. Belson, J. Thompson, J. Sun and R. Ericsson, "Q1 2017 State of the Internet / Connectivity Report," Akamai Technologies, Q1 2017. [Online]. Available: https://www.akamai.com/us/en/about/our-thinking/state-of-the-internetreport/global-state-of-the-internet-connectivity-reports.jsp.

[3] R. Boutaba, M. A. Salahuddin, N. Limam, S. Ayoubi, N. Shahriar, F. EstradaSolano and O. M. Caicedo, "A Comprehensive Survey on Machine Learning for Networking Evolution, Applications and Research Opportunities," Journal of Internet Services and Applications, vol. 9, no. 16, December 2018.

[4] Internet Assigned Numbers Authority, "Service Name and Transport Protocol Port Number Registry," Internet Assigned Numbers Authority, 123 2018. [Online]. Available: https://www.iana.org/assignments/service-names-port-numbers/servicenames-port-numbers.xhtml.

[5] OpenVPN Inc., "OpenVPN," OpenVPN Inc., 2018. [Online]. Available: https://openvpn.net/.

[6] SonicWall, "SonicWALL Solutions," SonicWall, 2018. [Online]. Available: https://www.sonicwall.com/en-us/support/knowledge-base/170505885674291.

[7] Ubiquiti Networks, Inc., "Ubiquiti Networks," Ubiquiti Networks, Inc., 2018. [Online]. Available: https://www.ubnt.com/. 
[8] nDPI, "Open and Extensible LGPLv3 Deep Packet Inspection Library," ntop, 2018. [Online]. Available: https://www.ntop.org/products/deep-packet-inspection/ndpi/.

[9] T. Karagiannis, K. Papagiannaki and M. Faloutsos, "BLINC: Multilevel Traffic Classification in the Dark," ACM SIGCOMM Comput Commun, vol. 4, p. 35, 2005.

[10] P. Bermolen, M. Mellia, M. Meo, D. Rossi and S. Valenti, "Abacus: Accurate behavioral classification of P2P-TV traffic," Elsevier B.V, vol. 12, no. 004, p. 18, 2010.

[11] A. Moore, D. Zuev and M. Crogan, "Discriminators for use in flow based classification," University of London, Department of Computer Science, p. 16, 2005.

[12] A. W. Moore and D. Zuev, "Internet Traffic Classification Using Bayesian Analysis Techniques," SIGMETRICS, p. 11, June 6, 2005.

[13] J. Ning, M. Yang, S. Cheng, Q. Dong and H. Xiong, "An Efficient SVM-based Method for Multi-Class Network Traffic Classification," IEEE, pp. 978-1-46730012, 2011.

[14] Stanford Vision Lab, "ImageNet," Stanford University, Princeton University, 2016. [Online]. Available: http://www.image-net.org/.

[15] A. Geiger, "The KITTI Vision Benchmark Suite," Karlsruhe Institute of Technology, Toyota Technological Institute at Chicago, 2018. [Online]. Available: http://www.cvlibs.net/datasets/kitti/. 
[16] K. Simonyan and A. Zisserman, "Very deep convolutional networks for large-scale image recognition," International Confrence on Learning Representations, pp. 114, April 10, 2015.

[17] L. Zhang, I. Saleh, S. Thapaliya, J. Louie, J. Figueroa-Hernandez and H. Ji, "An Empirical Evaluation of Machine Learning Appraoches for Species Identification Through Bioacoustics," Symposium on Signal \& Image Processing, Computer Vision \& Pattern Recognition, p. 6, 2017.

[18] Microsoft, "Skype," Microsoft, 2018. [Online]. Available: https://www.skype.com/en/.

[19] Cisco, "Webex," Cisco, 2018. [Online]. Available: https://www.webex.com/.

[20] LogMeIn, "Join.me," LogMeIn Inc., [Online]. Available: https://www.join.me/.

[21] Frostburn Studios, "Heroes of Newerth," Frostburn Studios, 2018. [Online]. Available: https://www.heroesofnewerth.com/.

[22] Valve Inc., "counter-strike.net," Valve Inc., 2018. [Online]. Available: counterstrike.net.

[23] Valve Inc., "DOTA 2," Valve Inc., 2018. [Online]. Available: https://www.dota2.com.

[24] Amazon, "Twitch.tv," Amazon, 2018. [Online]. Available: http://twitch.tv/.

[25] Netflix, Inc., "Netflix," Netflix, Inc., 2018. [Online]. Available: https://www.netflix.com/.

[26] Google, "Youtube," Google, 2018. [Online]. Available: https://www.youtube.com/. 
[27] Valve, "Steam," Valve, 2018. [Online]. Available: https://store.steampowered.com.

[28] Electronic Arts, "Origin," Electronic Arts, 2018. [Online]. Available: https://www.origin.com/.

[29] Blizzard Entertainment, "Battlenet," Blizzard Entertainment, 2018. [Online]. Available: https://www.blizzard.com/.

[30] Tixati Software Inc., "Tixati," Tixati Software Inc., 2018. [Online]. Available: https://www.tixati.com/.

[31] Bittorrent, "uTorrent," Bittorrent, 2018. [Online]. Available: https://www.utorrent.com.

[32] Rubicon Communications, LLC, "pfsense," Rubicon Communications, LLC, December 2018. [Online]. Available: https://www.pfsense.org/.

[33] TCPDump/LibPCap, "TCPDump \& LibPCap," TCPDump/LibPCap, 2018. [Online]. Available: http://www.tcpdump.org/.

[34] L. Chaorong, A. Ji and Z. Cao, "Stressed Fibonacci spiral patterns of definite chirality.," Applied Physics Letters 90, vol. 90, 2007.

[35] M. D. Zeiler, "ADADELTA: an adaptive learning rate method," arXiv, vol. 1, no. 1212.5701, pp. 1-6, December 2012.

[36] S. J. Judd, Neural Network Design and the Complexity of Learning, Pasadena: Computer Science Department California Institute of Technology, Sept. 1988.. 
[37] M. Crotti, M. Dusi, F. Gringoli and L. Salgarelli, "Traffic Classification through Simple Statistical Fingerprinting," ACMSIGCOMM Computer Communication Review, vol. 37, no. 1, 2007.

[38] N. Jing, Y. Ming, C. Shaoyin, Q. Dong and H. Xiong, "An Efficient SVM-based Method for Multi-class Network Traffic Classification," 30th IEEE International Performance Computing and Communications Conference, 2011.

[39] M. Roughan, S. Subhabrata, O. Spatscheck and N. Duffield, "Class-of-service Mapping for QoS," Proceedings of the 4th ACM SIGCOMM Conference on Internet Measurement, no. 4, April 2004.

[40] J. Parks, "All disk galaxies rotate once every billion years," Astronomy, 13 March 2018. [Online]. Available: http://www.astronomy.com/news/2018/03/all-galaxiesrotate-once-every-billion-years.

[41] Wikimedia, "Cumba Meillandina," Wikipedia, 14 February 2006. [Online]. Available: https://commons.wikimedia.org/wiki/File:Small_Red_Rose.JPG.

[42] M. Bradshaw and C. Bower, "A bird's-eye view," Townson University, 16 May 2018. [Online]. Available: https://www.towson.edu/news/2018/chrisbower.html.

[43] P. Wightman and N. Wightman, "2005 Trip to Costa Rica," mikebaird.com, 2005. [Online]. Available: http://mikebaird.com/CostaRica/2005/index.htm. 


\section{APPENDIX A - ACRONYMS}

- Mbps: Megabits per Second

- NTI: Network Traffic Image

- CNN: Convolutional Neural Network

- NN: Neural Network

- SVM: Support Vector Machine

- DNN: Deep Neural Network

- IANA: Internet Assigned Numbers Authority

- QoS: Quality of Service

- DPI: Deep Packet Inspection

- VoIP: Voice over Internet Protocol

- DoTA2: Defense of the Ancients 2

- CSGO: Counter Strike Global Offensive 